\title{
Gradhiva
}

GRADHIV

Revue d'anthropologie et d'histoire des arts

18 | 2013

Le monde selon l'Unesco

\section{Archéologie et ventriloquie. Jeux de chaises et de choses au bord d'une tranchée archéologique}

Archaeology and ventriloquism: making objects speak in an archaeological dig

\section{Emmanuel Grimaud}

\section{OpenEdition}

\section{Journals}

Édition électronique

URL : http://journals.openedition.org/gradhiva/2750

DOI : 10.4000/gradhiva.2750

ISSN : 1760-849X

Éditeur

Musée du quai Branly Jacques Chirac

\section{Édition imprimée}

Date de publication : 1 décembre 2013

Pagination : 200-233

ISBN : 978-2-35744-072-2

ISSN : 0764-8928

Référence électronique

Emmanuel Grimaud, «Archéologie et ventriloquie. Jeux de chaises et de choses au bord d'une tranchée archéologique », Gradhiva [En ligne], 18 | 2013, mis en ligne le 01 décembre 2016, consulté le 30 avril 2019. URL : http://journals.openedition.org/gradhiva/2750 ; DOI : 10.4000/gradhiva.2750 

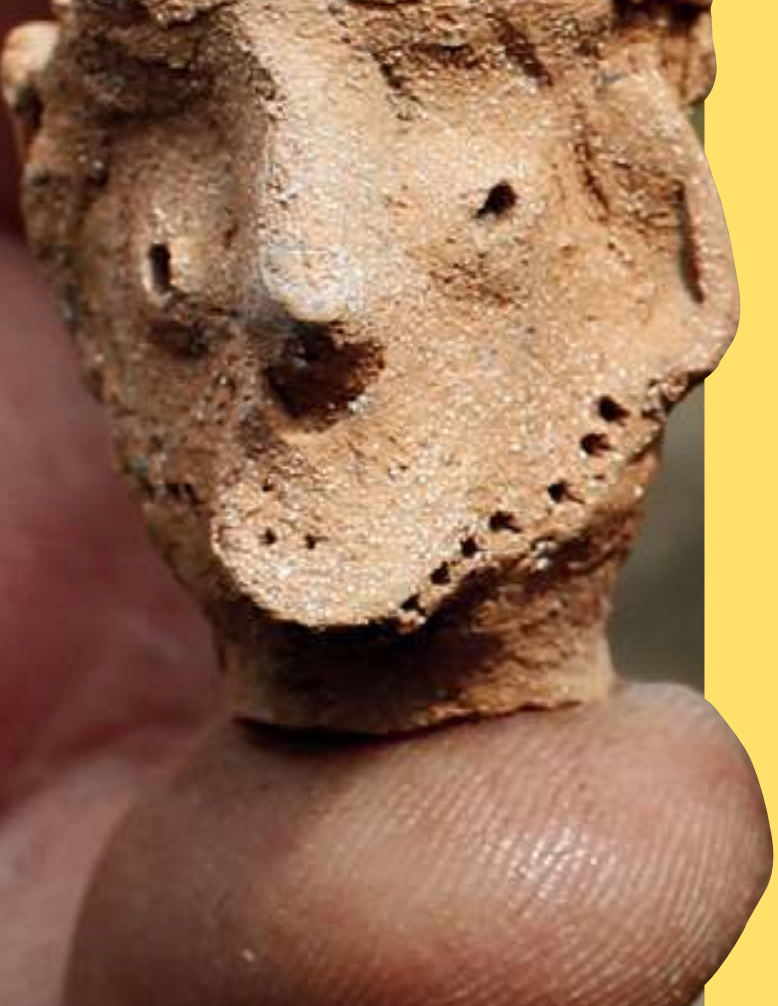

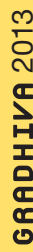

18 


\section{Archéologie et ventriloquie}

Jeux de chaises et de choses au bord d'une tranchée archéologique

par Emmanuel Grimaud

Une fouille archéologique est le terrain

d'expériences multiples de pensée,

«à l'extrême limite de l'identifiable, au presque

degré zéro de l'indice, à la frontière du rien » (Cohen 2011: 83). On tentera de mieux qualifier ces expériences de représentation à partir de quelques situations ethnographiques observées au cours d'une fouille indo-américaine en Inde. Au bord des tranchées, on s'adonne à des expériences ventriloques visant à faire parler des objets muets, à des expériences immersives (dans un monde révolu en trois dimensions), à des jeux de rôle (consistant à se mettre à la place de personnages ayant existé) ou à des expériences optiques sur des objets non identifiés et des figures fictives. Plus les situations limites aux frontières du savoir se multiplient, plus de nouvelles expériences, différentes des précédentes, doivent être tentées. Et plus les fouilles s'ouvrent au public et admettent de nouveaux intervenants, plus le sol devient une surface de projection singulière, une arène d'expression où les énigmes et les points de vue sont mis en partage dans des jeux interactifs dont cet article examine les modalités. 
1. François Cooren (2013) se sert de la métaphore ventriloque pour analyser, dans le cours même des interactions humaines, les multiples «manières de faire parler » les entités les plus diverses (institutions, valeurs, autres êtres, etc.). Renouvelant la pragmatique de la communication, il considère toute conversation comme le lieu d'une "dislocation de la parole " où ce qu'il appelle des «figures» se trouvent mobilisées et incarnées à différents degrés. Les interactants passent leur temps à parler en leur nom en même temps qu'ils sont mus par elles: "Toute communication, écrit-il, se retrouve, dans une certaine mesure, hors de son temps et hors de son lieu [...] parce que les interactants se trouvent, de façon hantée et fantomatique, à mettre en scène des figures venues d'ailleurs, qui viennent s'incarner/se représenter/ s'incorporer dans leurs interactions. " (Cooren 2013: 59)

2. Depuis les travaux de Carlo Ginzburg sur le paradigme indiciaire (2010 [1986]), les historiens et anthropologues savent à quelle branche du savoir raccrocher l'archéologie et ses méthodes complexes qui consistent à produire, à partir d'indices et d'inférences,

des scénarios plausibles. Le paradigme indiciaire s'est révélé fécond au-delà des frontières de l'historiographie pour comparer les méthodes d'enquête et les procédures de validation de la preuve dans des domaines aussi divers que la médecine, l'enquête policière, l'art ou encore la paléontologie et la divination. Voir sur ce point le volume coordonné par Denis Thouard (2007).
3. Il est utile de rappeler à quel point l'interprétation des indices a été perçue comme un mode de connaissance en soi où l'imagination, le flair et l'intuition jouent un rôle majeur. Lorsque Charles Sanders Peirce fait la distinction entre trois formes de raisonnement

- la déduction (la méthode des mathématiques),

l'induction (la méthode des sciences expérimentales) et l'abduction (entendue comme une inférence logique qui exprime sa conclusion de façon seulement problématique ou conjecturale) -, il insiste sur la troisième forme comme étant motrice de l'invention scientifique (Peirce 2002 [1903]). Sur la méthode de Zadig ou ce que Thomas Henry Huxley nommait "prophétie rétrospective " (1880), voir la très belle enquête historique de Claudine Cohen à propos de la paléontologie et de ses méthodes de reconstitution du «tissu du vivant » (2011).

4. Dans les deux dernières décennies, les pratiques archéologiques ont fait l'objet d'un intérêt croissant en anthropologie.

Les ethnographies se sont multipliées, des méthodes de fouille jusqu'à la gestion patrimoniale des collections en passant par les controverses sur l'identité des objets. L'archéologie dite "réflexive» ou "postprocessuelle» (Shanks 1992; Hodder 1999; Tilley 2004) a fortement encouragé ce mouvement, débouchant sur des tentatives très inégales de redescription sensible de l'expérience archéologique. II serait laborieux de citer tous ces travaux, mais on pourra consulter ceux de Matt Edgeworth (1992, 2003, [éd.] 2006, 2007). L'ethnographie de l'archéologie ne doit pas être confondue avec l'« ethnoarchéologie ", branche de l'archéologie qui prend pour objet des communautés vivantes afin de constituer des «référentiels" pour éclairer les pratiques du passé et élargir le spectre des plausibles (Binford 1980; Audouze et Perlès 1980).
"Ventriloquism is the occasion for letting strange voices speak. » David Goldblatt, Art and Ventriloquism, 2006

«[...] contemplant ces morceaux colorés de fresques romaines, tels qu'ils étaient là, insensés mais évidents. Je ne les regardais pas comme les vestiges d'un tout perdu mais comme l'affirmation d'un état possible et suffisant des choses, qui n'avait désormais plus de compte à rendre à un ordre antérieur. [...] Cette irréductibilité des fragments à réintégrer l'ensemble originel amorça, par le biais des lacunes, la disparition du support et la perte définitive du modèle, I'hypothèse d'une nouvelle redistribution du monde. » Emmanuel Hocquard, Une grammaire de Tanger, 2007

En quoi l'archéologie a-t-elle à voir avec la ventriloquie, cet art qui consiste à «faire parler des voix étranges", selon la formule de David Goldblatt? Les archéologues font parler les civilisations qu'ils exhument, et celles-ci leur font tout autant dire des choses qu'ils n'avaient pas même imaginées. La ventriloquie ainsi entendue ${ }^{\mathbf{1}}$ n'est pas propre à l'archéologie, elle imprègne l'ensemble des interactions humaines puisque nous passons notre temps à faire parler des entités qui ne sont pas là et qui parlent à travers nous (François Cooren dirait que nous sommes autant «ventriloqués» que nous nous faisons ventriloques). Ce problème de pragmatique de la communication est néanmoins particulièrement intéressant à observer sur une fouille, où des tas d'entités possédant des degrés de matérialité variables surgissent du sol, y compris des choses non identifiées ou «en attente d'étiquette ", stimulant de multiples jeux de parole.

Afin de se donner le maximum de matière, les archéologues pratiquent une forme de savoir indiciaire ${ }^{2}$ particulièrement éprouvante pour nos capacités d'inférence consistant à remonter, en différé, à partir d'indices, à des actes, des événements et des pratiques qui ne sont pas directement expérimentables ${ }^{3}$. Il leur faut mobiliser pour cela un savoir encyclopédique caché, des répertoires de formes, d'actions et d'images mentales aussi divers qu'il existe d'archéologies, voire de chantiers de fouilles. Toutes choses que les archéologues a priori savent déjà; la présence d'un anthropologue parmi eux à l'étape de la fouille ne leur apprendra rien de nouveau sur cet aspect de leur $\mathrm{art}^{4}$. Mais peut-on caractériser l'expérience archéologique plus finement que comme une tentative de connexion avec un passé lointain ou comme la mise à l'épreuve de l'imagination scientifique par une matérialité muette? C'est par l'ethnographie des manières de faire parler les choses et de les animer ainsi que par celle des expériences de pensée $\mathbf{5}$ auxquelles les archéologues s'adonnent volontiers au cours de la fouille que j'espère arriver à éviter certaines apories des approches anthropologiques “critiques» de l'archéologie. Celles-ci ont en effet souvent du mal à proposer aux archéologues autre chose qu'une mise à distance de leurs pratiques de scénarisation, envisagées sous l'angle de l'imaginaire ou de la fiction ${ }^{6}$.

On s'intéressera ici autant à l'inventivité dont les archéologues témoignent pour débusquer de la présence humaine derrière les choses qu'aux difficultés de représentation auxquelles ils sont confrontés et qui 
les obligent à multiplier les expériences acrobatiques de pensée. II serait faux de croire qu'on peut faire dire n'importe quoi aux objets, comme le soulignent les dénonciateurs des «fictions" archéologiques (et parfois les archéologues eux-mêmes critiquant les hypothèses de leurs collègues ${ }^{7}$ ). Parce que les archéologues doivent s'immerger dans des espaces non identifiés ou instables, la fouille est propice à des expériences de représentation variées, «à l'extrême limite de l'identifiable, au presque degré zéro de l'indice, à la frontière du rien" (Cohen 2011: 83). Ces expériences de dislocation des conditions normales de la représentation engagent la pensée et l'imagination, mais aussi la vision et le toucher. Elles méritent d'être explorées de plus près car ce n'est qu'à cette condition, parce que le régime ordinaire de la représentation se trouve déstabilisé, que des entités très diverses par leur degré d'incarnation apparaissent, stimulant des formes ventriloques d'expression particulièrement délicates. L'hypothèse que nous proposons est que la plupart des situations de fouille où les traits du sol sont interrogés gagnent à être envisagées comme des exercices de contorsion de nature cinématographique où le réalisateur, les acteurs et les spectateurs intervertissent volontiers leurs positions. Parce que la fouille génère des difficultés de représentation et de visualisation, les archéologues sont contraints d'adopter face à ces traits des positions variables, multipliant les points de vue avant de se prononcer. Ils ne sont jamais simplement réductibles à des «interprètes» faisant parler des «objets». Au cours de la fouille, les rôles semblent se redistribuer continuellement entre l'écran, le réalisateur, l'acteur et le spectateur. Les scénarios s'effritent ou au contraire s'épaississent, au gré de nouvelles présences qu'on n'avait pas envisagées. Même si elle a certainement ses limites, la métaphore cinématographique dont usent parfois, on le verra, certains archéologues, permettra de mieux coller aux actes de regard et prises de parole dont on cherchera à saisir la dimension ventriloque.

\section{Indice et action}

L'archéologie est peut-être autant affaire de cinéma que de ventriloquie. Le fouilleur doit en effet suspecter le mouvement derrière l'inerte car une trace est toujours l'indice d'un bougé ou d'une action à laquelle on peut potentiellement remonter. Ainsi, l'un des premiers exercices de contorsion de la perception auquel un fouilleur se trouve confronté touche au rapport entre l'inerte et l'animé. Pourtant, la notion d'animation apparaît beaucoup moins discutée en archéologie que celle, tout aussi cinématographique, de scénario ${ }^{8}$. Ce dont rêve l'archéologue, c'est de pouvoir reconstituer un film de la civilisation qui l'occupe qui soit aussi animé qu'un vrai film. S'il y a un art qui a poussé très loin la technique de l'animation (image par image), jusqu'à en faire un métier, c'est bien le cinéma. Et s'il y a une science qui a cherché à faire un usage à la fois méthodologique et contrôlé de l'animation (indice après indice) et de l'interpolation ${ }^{9}$, mais sans caméra cette fois, c'est bien l'archéologie. Dès qu'elle démarre, une fouille génère d'innombrables jeux de décryptage et une production quasi ininterrompue de scénarios provisoires (des «hypothèses de travail») qui seront validés ou bien infirmés aux couches suivantes. Tout au long de la fouille, les archéologues cherchent à donner sens à ce qu'ils voient, ils se convoquent les uns les autres, ils partagent des hypothèses sur le bord de leurs tranchées et parfois avec les visiteurs de passage.
5. On ne peut rentrer

ici dans les nombreux débats que cette notion a provoqués en philosophie de la connaissance. Depuis les paradoxes de Zénon jusqu'à l'expérience dite du "cerveau dans une cuve» d'Hillary Putnam (1981) en passant par le bateau de Galilée, les expériences de pensée partent généralement d'une formule simple: "Que se passerait-il si...? "Sur les chantiers de fouilles, elles peuvent prendre des formes très variées. II ne s'agit pas d'en faire ici la liste ni même de proposer une typologie. Les exemples abordés dans la suite de cet article ne sont que des cas parmi bien d'autres.

6. On peut consulter ici l'ouvrage dirigé par Claudie Voisinat, Imaginaires archéologiques (2008), ainsi que notre compte rendu dans Gradhiva 9 (2009). Les archéologues eux-mêmes cultivent l'image de la fouille comme une épreuve de confrontation entre l'imagination et la matière lorsqu'ils distinguent la démarche hypothéticodéductive (du modèle aux données) et empirique (des données au modèle). Claudine Cohen suggère une autre voie possible Iorsqu'elle écrit, à propos des paléo-ichnologues qu'elle compare à des "Sherlock Holmes des temps modernes": "C'est alors toute la panoplie conceptuelle d'une science qui se déploie, c'est l'inventivité et l'imagination des chercheurs qui sont requises pour interpréter des "faits" qui se situent à l'extrême limite de l'identifiable [...]. » (Cohen 2011 : 83) De même, lorsqu'elle pointe l'" heuristique des faux " en préhistoire pour matérialiser des hypothèses scientifiques, elle montre comment, dans certains cas, la rareté des données oblige à déployer des trésors d'imagination méthodologique et conceptuelle.
7. Voir sur ce point les nombreuses critiques par les archéologues eux-mêmes des appropriations politiques de l'archéologie où le terme de «fiction » apparaît mobilisé de manière courante (Shanks 1992 ; Tilley 2004).

8. Sur la diversité des conceptions du scénario au cinéma, je me permets de renvoyer à Grimaud (2008).

9. Dans le cinéma d'animation, l'interpolation désigne un procédé permettant de générer des images intermédiaires successives afin que l'animation s'enchaîne de façon fluide. Sur l'histoire de ce procédé, voir le très beau livre de Dominique Willoughby (2008). 


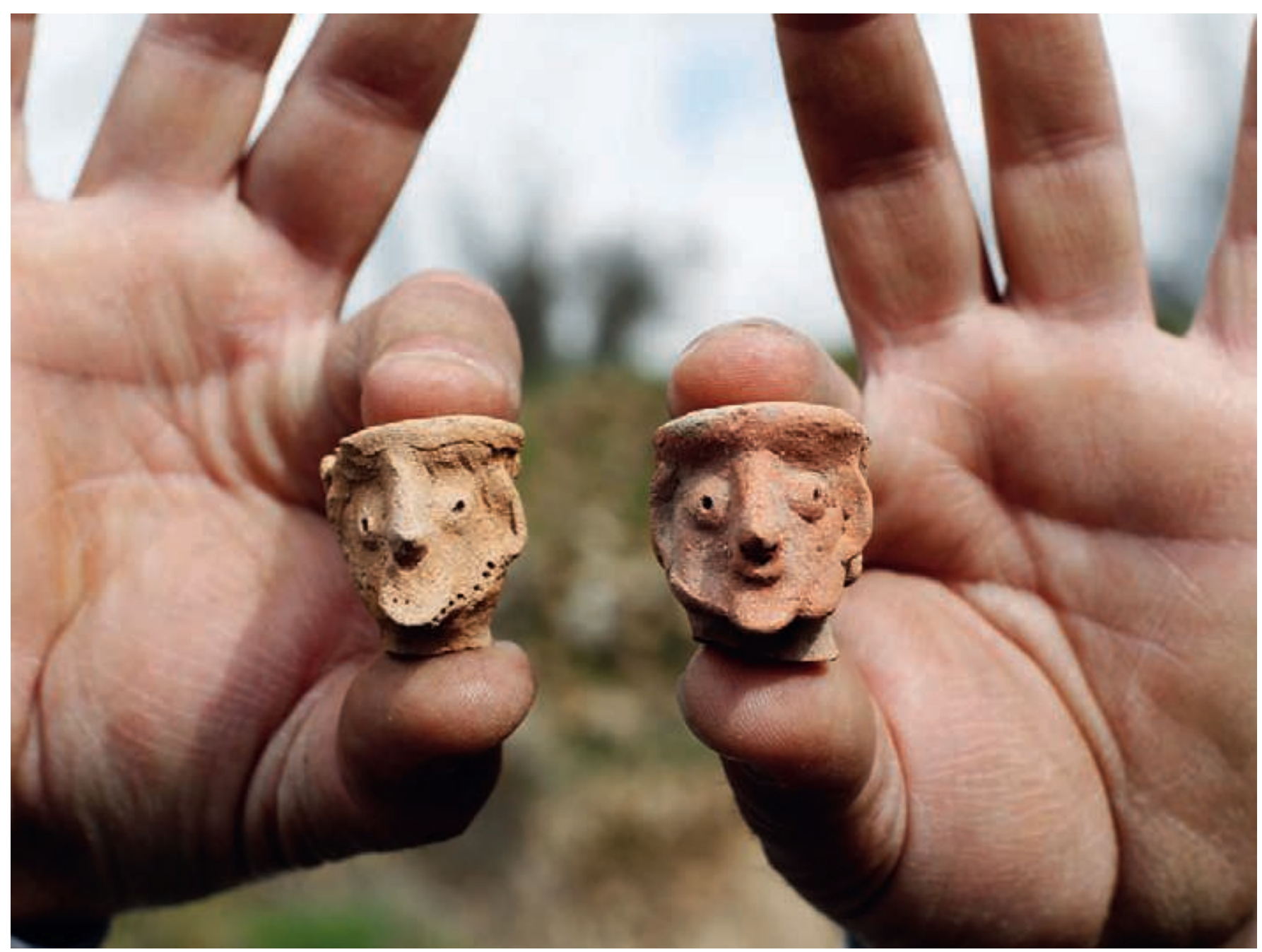

fig. 1

Employé des antiquités

israéliennes (Israel's Antiquities

Authority, IAA) montrant

une figurine du site archéologique

de Tel Motza près de Jérusalem,

2012. (C) REUTERS/Baz Ratner. 
Lorsque les fouilleurs s'expriment, ils font l'aller et retour du solide au vivant et inversement. Leurs conversations souvent animées visent à s'accorder sur de petits films d'action qui «incarnent» les indices perçus en fonction des présences qui les ont produites. Ce travail d'animation est d'autant plus intense que les choses exhumées sont incomplètes ou difficiles à interpréter. On a l'impression d'être sur une fouille comme sur le tournage d'un film qui aurait du mal à avancer par manque de décors ou d'acteurs, où les cinéastes ne cesseraient de refaire les scènes dans leur tête, de discuter d'options alternatives, ce remontage permanent se prolongeant bien après que la fouille est terminée. C'est que les fouilles archéologiques tout comme les tournages de films sont des formes d'expérience virtuelle qui impliquent l'immersion dans un monde. Ceux qui y participent basculent, avec une intensité variable selon leur engagement, dans une sorte de «matrice» plus ou moins palpable et opaque, qui peine parfois à s'actualiser soit parce que les éléments pour l'alimenter sont peu nombreux comme c'est le cas sur une fouille, soit en raison d'intempéries diverses ou par manque d'argent comme il arrive souvent sur un tournage.

Tentons de pousser un peu plus loin le parallèle et voyons où il nous mène: si la fouille met en acte une sorte de cinéma, de quel genre de cinéma s'agit-il et où devrions-nous situer l'écran et le projecteur? Si les archéologues, tout en fouillant, basculent dans une sorte de matrice virtuelle, comment rendre compte de cette expérience d'immersion sans succomber aux facilités des analyses en termes d'imaginaire? Le point commun entre le travail de fouille archéologique et l'expérience cinématographique ne peut être ni dans la nature de l'objet ni dans celle de l'image, car l'univers des archéologues n'est pas constitué d'images ou pas immédiatement, mais de matières friables et d'indices. Un producteur peut potentiellement ériger n'importe quel décor, même le plus fantaisiste, et un réalisateur et des acteurs incarner n'importe quelle histoire. Mais les archéologues, eux, doivent se contenter de ce qu'ils trouvent. II se pourrait bien qu'on ait intérêt à penser la caméra comme étant manipulée ici non par un être humain, mais par les choses elles-mêmes. Ce serait alors un genre de cinéma totalement inédit, un cinéma de terre ou de poussière, tactile avant d'être optique, un cinéma solide ${ }^{10}$ qui, tout en se passant d'écran et de projecteur, en aurait néanmoins tous les ingrédients. Postuler l'existence de personnages, d'actions et de scénarios («comme au cinéma») serait alors la manière la plus simple pour l'archéologue de «déchosifier» la matière inerte et de s'immerger dans l'espace plausible, en trois dimensions, d'une civilisation avec laquelle il ne peut établir le contact que sur un mode fragmentaire et intermittent.

\section{"Se mettre dans la peau d'un personnage qui...": la fouille comme expérience d'incorporation}

Une conversation avec un archéologue, couché au fond d'un trou qu'il pensait être une sépulture, nous renseignera sur la singularité de l'exercice archéologique comme «cinéma solide». Creusant avec sa truelle, la tête au fond d'un trou où il dépoussière scrupuleusement quelques débris d'ossements, le fouilleur me tient les propos suivants:

Tu as vu Memento, le film? C'est I'histoire d'un type qui devient amnésique à la suite d'un choc et essaie de se rappeler tout ce qui
10. L'expression n'est pas de moi mais de Paul Mus (1964). Les objets qui intéressaient Mus n'étaient pas les paysages archéologiques, mais les divinités décuplées de l'art indien classique qu'il comparait à un certain procédé utilisé dans les œuvres d'art cubistes, qui combinaient dans un même objet différentes prises de vue "chronophotographiques" de l'objet. C'est ainsi que les sculptures aux bras décuplés assemblaient selon lui les différents points de vue qu'un hindou pouvait avoir de la divinité lorsqu'il en faisait le tour lors de la circumambulation rituelle. 
s'est passé auparavant, il poursuit l'assassin de sa femme, je crois, et le film est fait de telle sorte qu'il va en arrière: ça commence par la fin, et ensuite tu as la séquence d'avant. Tu vois le personnage se réveiller dans un hôtel sans savoir où il est, et le spectateur ne le sait pas non plus. C'est toute une série de petites séquences qui vont en sens inverse du scénario, ce qui fait que tu te retrouves dans la peau d'un personnage qui voit d'autres personnages arriver sans savoir qui ils sont. Et petit à petit, tu entrecroises les trucs, c'est-àdire qu'on commence par la fin, on n'a pas tous les antécédents, on n'a pas tout le film. Là, je comprends pas ce qui se passe. [...] J'ai des petites pierres comme ça et des bouts d'os, donc j'arrive à proximité de quelque chose. Ce qui m'inquiète, c'est que... est-ce que je suis dans le remblai ou déjà dans la structure, c'est-à-dire plus bas, déjà? Je vais voir en continuant... en continuant rétrospectivement. Ces pierres-là, j'ai l'impression que ce ne sont pas les mêmes que précédemment. Mais après tous les indices qu'on a eus jusqu'à présent, et qui vont tous dans le sens d'une sépulture, ce serait étonnant qu'il n'y ait rien du tout...

Etre dans la peau d'un personnage qui voit d'autres personnages arriver sans savoir qui ils sont. Voilà qui résume bien l'impression, que peut parfois éprouver l'archéologue, d'être manipulé par les choses qu'il exhume. À la fois acteur et spectateur dans sa propre tranchée, le fouilleur se retrouve soudain mis en présence d'un autre (le squelette). Cette présence acquiert ici suffisamment de matérialité pour qu'il se croie pris dans le même film que ces présences qu'il exhume et dont le réalisateur est bien sûr inconnu. C'est par un effort de redescription sensible, aussi fidèle que possible à ces contorsions des conditions même de la représentation, qu'on s'aperçoit que le processus archéologique n'oscille jamais entre le réel et la fiction, mais plutôt entre quatre dimensions: par la fouille, une surface plane en deux dimensions devient un espace immersif en trois dimensions puis un espace stratifié à quatre dimensions. Les jeux d'animation qui s'instaurent entre archéologues sur le bord des tranchées visent à explorer les possibles en changeant alors plusieurs fois de position (acteur, spectateur, personnage). Ce n'est qu'ainsi qu'on arrive à confirmer le type d'espace auquel on a affaire, son évolution, et à trancher entre un grand nombre d'impossibles et un petit nombre de plausibles. En Inde, où j'ai travaillé en tant que trench supervisor, j'ai été frappé de constater que les tranchées de fouilles étaient entièrement accessibles au public. Visiteurs, archéologues ou simples spectateurs se succédaient et participaient activement à ces jeux d'animation. Dans ce grand puzzle à ciel ouvert que constituait la fouille, les gens s'asseyaient et tentaient de ramener les traits du sol à des événements ou d'établir des connexions entre les formes qu'ils distinguaient. On reviendra plus loin sur cet élargissement du jeu interprétatif, mais commençons par des interactions simples, qui interviennent lorsque le fouilleur en pleine action gratte le sol avec sa truelle. On y verra apparaître toutes les difficultés à faire d'une surface plane l'objet d'une expérience d'immersion dans un espace en trois dimensions. De cette tension naissent les énigmes, les plus petites, qui sont le lot des fouilles ordinaires, comme les plus grandes, qui se muent en controverses. 


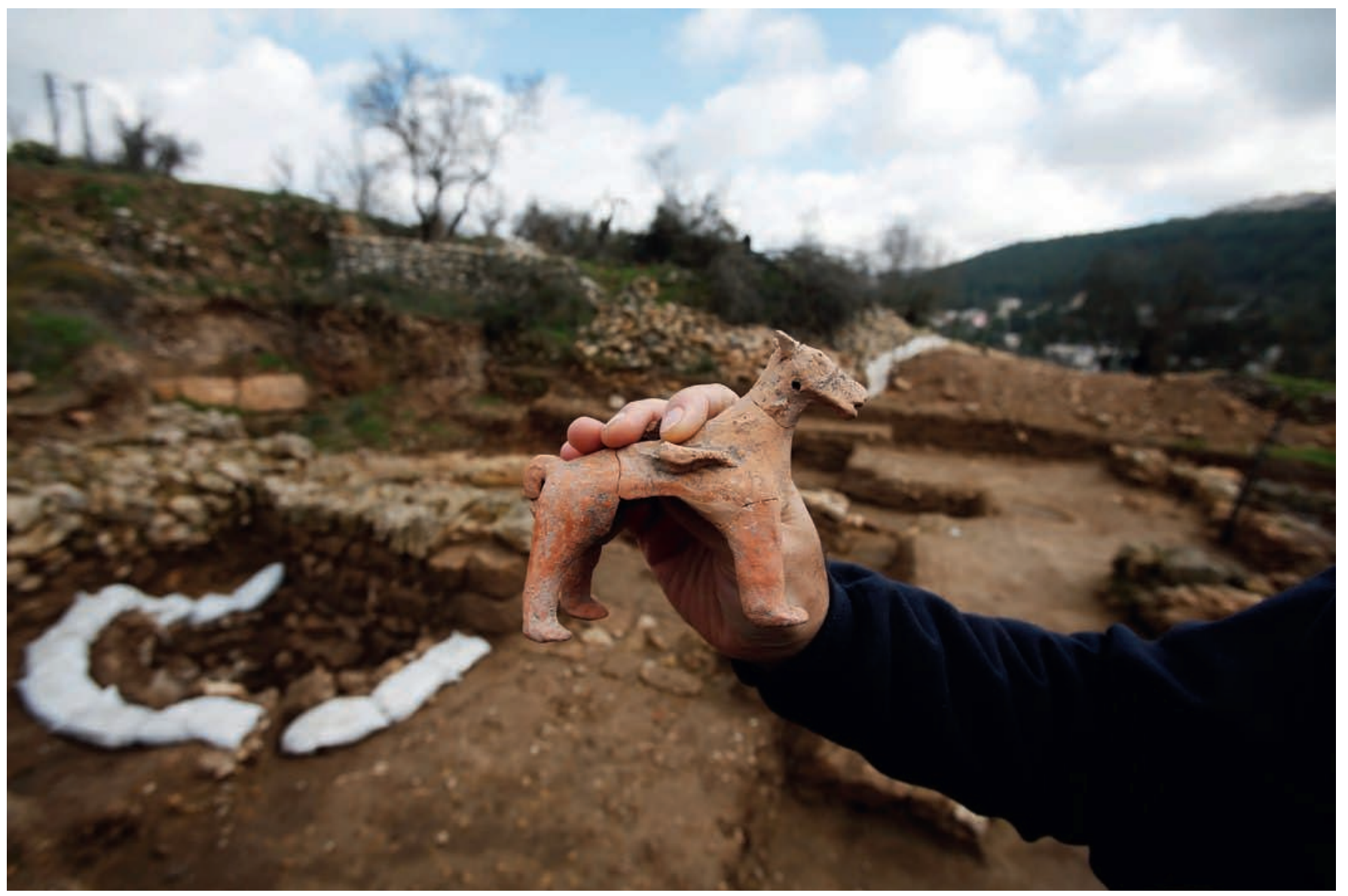

fig. 2

L'archéologue Anna Eirikh avec une figurine de cheval provenant du site

de Tel Motza, Israël, 2012.

(C) REUTERS/Baz Ratner. 


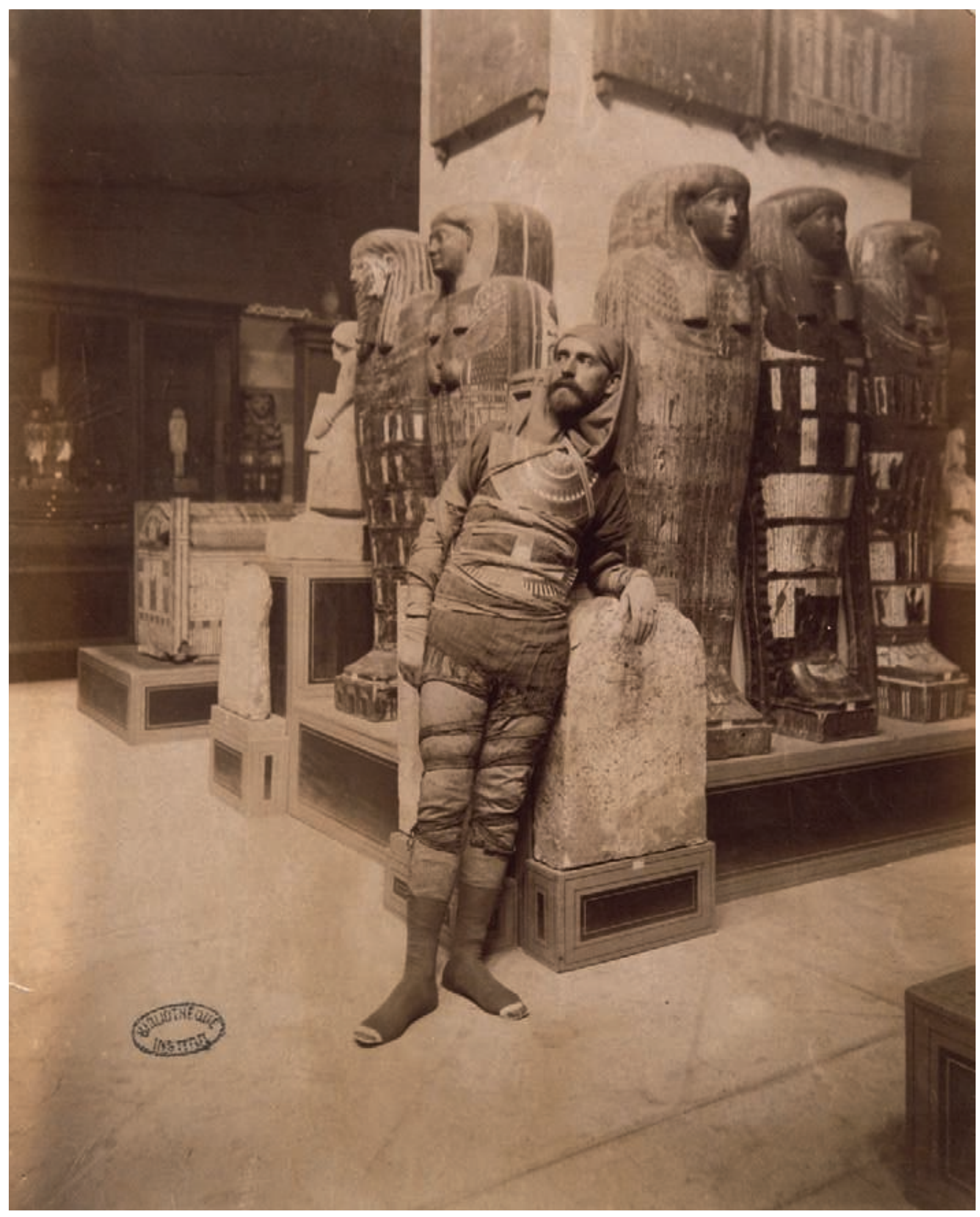


"Faire comme si nous étions dans...": une expérience de fouille à l'aveugle

Nous sommes sur une fouille indo-américaine au Rajasthan. Le DrP., un archéologue américain bien connu, s'assied au bord de la tranchée sur son siège pliant. C'est sans doute l'une de ses dernières fouilles en Inde car il est désormais âgé, mais les indices d'une microculture dans un rapport ambigu à la civilisation de l'Indus l'ont motivé à ne pas abandonner le métier. II fouille cette fois avec le Dr S., un archéologue indien réputé. Les deux hommes ont des méthodes et des vues souvent divergentes (méthode horizontale chez les Américains, verticale pour les Indiens), mais pas toujours ${ }^{\mathbf{1 1}}$. Ils sont d'accord, par exemple, sur le fait qu'il faut tirer partie de l'occasion de la fouille pour faire de la formation, initier les étudiants américains aux singularités du sol indien, les accompagner dans ce premier contact, et former les étudiants indiens à des méthodes de fouille plus horizontales que celles, stratigraphiques, d'ordinaire utilisées par les archéologues locaux. Derrière le Dr P., son vieil assistant, un habitant du village qui le suit partout, tient un parapluie pour le protéger du soleil. Le Dr P. est connu pour être un remarquable professeur. II guide ici la truelle de son étudiante, responsable d'un carré de fouille. Celle-ci est penchée au ras du sol et gratte la terre. Elle semble avoir exhumé certains «traits» - un pot, quelque chose qui ressemble à un four et une «petite chose noire» - qui apparaissent particulièrement signifiants à l'archéologue assis sur sa chaise pliante:

- Je veux que tu n'enlèves rien de tout ça, tu nettoies, tu dépoussières, c'est tout!

- Non, je ne retire rien...

- C'est important de le garder.

$-[\ldots]$

- Je veux que le Dr S. voie ça, il vous dira quoi faire et comment. Mais je te dis ce que je ferais à ta place, je le laisserais en place. Tu sais, le pot est très en surface, il est encastré là, ce four est imbriqué comme ça, mais il ne l'était pas avant probablement, donc ma stratégie, ce serait d'essayer de voir ce qu'il y autour du pot et de comprendre ce qui lui est associé. Le four ne lui est pas associé. Mais ne touche pas au four, si tu le retires, il ne reviendra jamais. La seule raison pour laquelle je l'enlèverais serait pour retracer la relation entre ici et là.

Qu'est-ce que tu penses de la relation entre ça et les fragments de pot que tu viens de faire apparaître?

L'étudiante continue à gratter avec la truelle et dépoussiérer avec la brosse.

Quelques minutes plus tard...

- Tu as un autre pot, semble-t-il?

- Oui!

- Qu'est-ce qu'il y a de l'autre côté? [...] C'est quoi ce motif noir, c'est un dessin?

Le Dr P. pointe la zone avec son mètre.

- Je veux que tu m'exposes ça, mais tu n'enlèves rien! Est-ce qu'il y a un autre pot par ici?

- Je ne sais pas, je gratte encore... J'enlève ça?

- Oui, vas-y! Ne le retire pas si ça résiste trop. [Elle abandonne la truelle

11. Les controverses publiques de l'archéologie indienne, et notamment autour de la civilisation de l'Indus, ont largement intéressé les historiens et les sociologues (Bryant et Patton [éd.] 2005 ; Guha 2005). L'archéologie indienne offre un terrain remarquablement propice à des formes très diverses et mal étudiées d'«imagination cryptographique", pour reprendre la belle formule d'Ashis Chadha (2010).

\section{ci-contre}

fig. 3

Eduardo Toda y Gu'll

en momie, photographié au musée de Boulak (préfiguration du musée du Caire), 1886. Bibliothèque de l'Institut, Fonds Maspero. (c) RMN-Grand Palais (Institut de France) / Gérard Blot. 
12. Sur l'acte de pointer en situation de fouille et la nécessité d'orienter l'attention dans des configurations de stimuli hétérogènes, voir le travail de Charles Goodwin (2003). pour la brosse.] O.K., maintenant, essaie d'enlever tout ce dépôt ici, autour du morceau calciné. Toujours du pot vers l'extérieur, du connu vers l'inconnu. C'est tout, ça suffit. Il est 5 heures, protège-le bien avec un seau par-dessus et du remblai. Personne ne doit y toucher. Cette chose noire est importante. Sors de ton trou, viens voir depuis le bord ce que ça donne. [Elle sort du trou.] Maintenant, que vois-tu?

- Cela ressemble à...

- Peu importe ce que c'est, c'est important de le protéger! Ce n'est peut-être rien du tout, mais c'est mieux de ne pas déranger la chose! L'archéologue s'en va et laisse l'étudiante clore sa tranchée pour la nuit. La «chose» non identifiée peut dormir tranquille. L'étudiante du Dr $P$. est fatiguée. Les yeux pleins de poussière, elle dit:

- On n'y voit plus rien ici, c'est terrible, on a du mal à faire la différence entre la brique crue et la terre!

Toute la difficulté pour le Dr P. est de montrer à son apprentie comment faire "comme si» elle était dans un espace de vie possible, à l'intérieur d'une maison ou dans un atelier d'artisan, c'est-à-dire un espace en trois dimensions, alors qu'elle a le nez collé au sol. Il faut qu'elle s'habitue à travailler ainsi avec des présences absentes ou qu'elle soit toujours prête à voir surgir de nouvelles présences. Mais on retrouve ici le même effet que dans le cas précédent de déstabilisation du régime normal de la représentation, voire de mise au défi des conditions d'exercice de la perception («on n'y voit plus rien»). Où sommes-nous? La question reste entière jusqu'au bout. D'où l'importance de guider la truelle et la brosse de la fouilleuse à distance. Le Dr P. a le regard rivé sur le moindre de ses changements de direction ou de pression. Il lui dit où aller, l'oriente et indique le degré de force ou de délicatesse à employer. L'archéologue instaure avec la truelle de son étudiante une relation de commande. II n'est pas comme un réalisateur guidant son actrice pour qu'elle incarne au mieux le scénario qu'il a en tête, mais plutôt comme un spectateur qui voudrait voir un certain type de film avec un maximum d'objets inédits ou non identifiés. L'étudiante a incorporé le désir de son professeur de faire parler les choses et teste la capacité de ces mêmes choses à parler. Actrice dans sa propre tranchée mais pas seule spectatrice, elle est dans une situation de ventriloquie particulièrement compliquée, avec d'un côté un spectateur très présent, demandeur d'un certain type de scénario et qui «surveille» ses faits et gestes, et de l'autre des personnages qui n'ont même pas encore surgi, mais dont elle doit postuler l'existence et qui devraient idéalement s'exprimer à travers sa truelle.

Et quand tu as affaire à des briques désorganisées, lui dit le professeur, enlève d'abord les briques qui sont en désordre, fais une hypothèse quant à leur orientation la plus probable, enlève tout ce qui va à l'encontre de cette orientation. C'est le meilleur moyen de ne pas faire d'erreur.

La fouilleuse sort éprouvée physiquement et mentalement de l'expérience. II ne s'agit pas seulement d'être capable de pointer les bons indices ${ }^{\mathbf{1 2}}$. Le Dr P. tente de l'initier à autre chose, à une forme de rapport de forces avec la matière car il est facile de s'y perdre, de s'oublier. II faut que la stratégie reprenne le dessus, qu'une forme de conceptualisation active s'invite dans l'acte de maniement de la truelle. Grâce au Dr P., la fouilleuse intègre 
ici quelques principes de pensée tactile bien utiles. Elle se laisse guider par des zones de tact, de contact, de frottement, d'effritement, des rapports de friction et de résistance. Certains objets s'effritent ou disparaissent aussitôt effleurés; le fouilleur détruit beaucoup parce qu'il espère trouver mieux plus bas, qu'il ne voit pas ou encore qu'il n'établit aucun lien entre les éléments en présence. II faut se ménager l'espace d'une attribution possible, mais cet espace n'est pas donné d'emblée, il se conquiert. Rares en effet sont les situations claires où l'on peut se dire, comme dans l'expérience de Fritz Heider et Marianne Simmel ${ }^{\mathbf{1 3}}$ (1944), que la tranchée est simplement formée de deux triangles, d'un rond et d'un carré. Très vite, son paysage se modifie, les objets non identifiés se multiplient, il faut revoir sa stratégie, faire des choix et peut-être se raccrocher à des principes simples («aller du connu vers l'inconnu» par exemple). L'archéologue expérimenté pratique des touchers adaptés sans s'en apercevoir, quasi immédiatement, exerçant en quelque sorte son habitus archéologique. II suffit d'attaquer à la pioche un petit tas de matière friable pour se rendre compte de la nécessité d'acquérir une juste appréhension des résistances. C'est d'ailleurs un autre principe que j'ai souvent entendu dans la bouche du Dr P.: «Être suffisamment prudent pour ne rien détruire et suffisamment agressif pour tester ses hypothèses. 》

En voulant protéger la «petite chose noire» qu'il ne faut pas déranger au risque de la détruire, le Dr P. attire notre attention sur un problème familier des psychologues de l'attribution. Il engage sa fouilleuse dans une forme d'exercice perceptif comparable à l'expérience de Heidel et Simmel, qui présentaient des figures géométriques animées à un public en leur disant: «Décrivez l'action, quel drame voyez-vous? " Le Dr P. dit à sa fouilleuse: «Attention, il y a peut-être là du drame, matière à un scénario. » Mais ce qui permet ici d'amorcer le scénario n'est pas à proprement parler d'ordre narratif. Quand on est à l'affût de ce qui peut s'exprimer au ras du sol, un trait non identifiable mais suffisamment distinct de l'arrière-plan suffit à catalyser de la présence et à stimuler des attributions. Deux composants non identifiés peuvent mieux encore déclencher dans le cerveau du fouilleur un ensemble de mécanismes visant à déterminer s'ils sont liés ou non et, si oui, dans quel rapport ils se trouvent. II faut peu de chose pour faire jaillir des fantômes, il suffit d'une répartition de différences sur un plan pour que l'on s'interroge sur leurs rapports et que «quelqu'un » ou « une autre action » en soit le lien. Plus la tranchée se peuple de telles «choses» qui, sans être à proprement parler identifiables, sont reconnues comme des indices de présence humaine, plus on laisse le champ libre à des expériences d'incorporation comme celle que l'on a vue précédemment, où le fouilleur ne gratte pas seul mais «en présence» de personnages qui l'ont précédé et auxquels il doit un minimum s'identifier.

\section{"Faire face à un tas, le traverser et...":} déambulation dans un espace dévitalisé

Suivons à nouveau le Dr P. qui va nous introduire à d'autres problèmes de représentation. II emmène ses fouilleurs pour se faire l'œil dans le village d'à côté. II s'est aperçu que ses étudiants, américains en particulier, avaient du mal à se représenter certaines formes basiques que l'on retrouve communément dans le sol indien: un four, une maison, etc. S'ils voulaient se donner les chances de voir surgir des entités plausibles,
13. Les psychologues Fritz Heider et Marianne Simmel montrèrent à diverses assemblées un film d'animation très court, où deux triangles et un rond bougeaient à l'intérieur et à l'extérieur d'un carré qui s'ouvrait et se refermait. Durant la projection, le public ne pouvait s'empêcher d'attribuer à ces figures géométriques un comportement, une intentionnalité. L'un des triangles était jugé particulièrement "agressif », " excité " ou «insistant ", tandis que le rond pouvait paraître «fragile» ou "hésitant". Certains disaient que «Mme Triangle cherchait à protéger son petit de M. Triangle qui finissait par détruire la maison [le rectangle] ", ou encore que «Papa Triangle était en concurrence avec maman Triangle ", etc. L'une des principales qualités du film est de déclencher chez celui qui le voit un processus dynamique de recherche de causes et de motivations. Le scénario élaboré varie ensuite d'une séquence à l'autre, selon les figures auxquelles l'origine de l'action est attribuée et selon que l'on perçoit l'action d'abord et les figures ensuite ou l'inverse. 
il leur serait utile de s'imprégner de toutes ces formes dans le village voisin. C'est un peu comme si Heider et Simmel, à force de se confronter au même scénario œdipien sur leurs petites figures, avaient décidé de s'aventurer dans une tout autre entreprise: élargir l'univers de références de leurs sujets pour leur montrer qu'un trio n'est pas forcément un western ou ne compose pas nécessairement une famille. C'est que les fouilleurs sont moins libres d'établir des connexions qu'il n'y paraît car les choses sont paradoxalement plus imprévisibles, forçant les humains au terrible exercice de deviner ce qui les unit, mettant leurs mécanismes d'attribution à rude épreuve. Les fouilleurs pourraient bien être, au fond de leur tranchée, comme le public du film de Heider et Simmel qui passait des figures à leurs actions, établissant des liens, attribuant des motivations à des figures à peine lisibles, courant après l'intention d'un cinéaste disparu. Une tranchée archéologique ne serait alors pas si éloignée, en termes d'attribution causale, d'un film d'action bourré d'ellipses ou d'un dessin animé dont on n'aurait plus qu'une image sur trois. L'intérêt de la visite qui va suivre est de donner aux fouilleurs des repères pour passer en cours de fouille, autant de fois que nécessaire, d'un espace vivant à un espace dévitalisé. Ils seront alors prêts à effectuer le grand saut dans une civilisation vieille de plusieurs millénaires.

«Regardez-moi tout ça!» Le Dr P. pointe du doigt quelques tas. On dirait que le village n'est fait que de ça: des tas de pierres, des tas de briques posés en travers de la route. "Qu'est-ce que cela veut dire? » leur demande-t-il. «Pourquoi est-ce au milieu de la rue? Ça ne vous étonne pas? Interrogez-vous sur l'intention qu'il y a derrière... Et regardez-moi cette maison, toutes ces planches posées sur le bord des façades. À votre avis, est-ce la maison d'un charpentier ou une maison en construction? Et voyez ce puits, cela coûte beaucoup d'argent de faire ce genre de chose. Observez bien sa localisation... » Devant une petite échoppe de thé, le Dr P. ne s'intéresse pas aux gens qui le préparent mais au four et à la manière dont il est disposé. Il explique le processus d'allumage et d'entretien du feu. «Vous pouvez trouver ce genre de four sous la terre, c'est pourquoi je vous demande de bien le regarder. " Le Dr P. n'est pas étonné par la couleur des saris des femmes ou les colliers des vaches, il va généralement au moins spectaculaire, à ce qui structure l'espace. Ainsi, il est intrigué par un puits situé à un carrefour de ruelles bien plus larges que d'ordinaire. II faut que les troupeaux puissent y accéder, que les femmes puissent porter l'eau dans des pots. «Pensez-vous que le puits a été fait avant les routes ou après? Lequel des deux précède l'autre, le carrefour ou le puits? C'est ce genre de question qu'il faut vous poser. »

Plus loin, le Dr P. s'arrête devant un tas d'excréments. «Ce n'est pas de très bon goût, mais je tiens à m'y arrêter, c'est ici que les gens viennent faire leurs besoins, ils ne vont pas forcément dans les champs. Tenezvous prêts! » II fallait donner aux fouilleurs des images de référence car ils pourraient bien tomber sur des scénarios similaires une fois dans leur trou. Le Dr P. ne dit pas: “C'est ce que vous allez trouver parce que les gens continuent à faire la même chose depuis des millénaires et qu'on se situe dans la même région. " II dit plutôt: «Regardez comme c'est étrange de manquer d'informations pour connecter entre elles certaines choses alors que vous croyez avoir tous les éléments devant les yeux. " II s'arrête au 


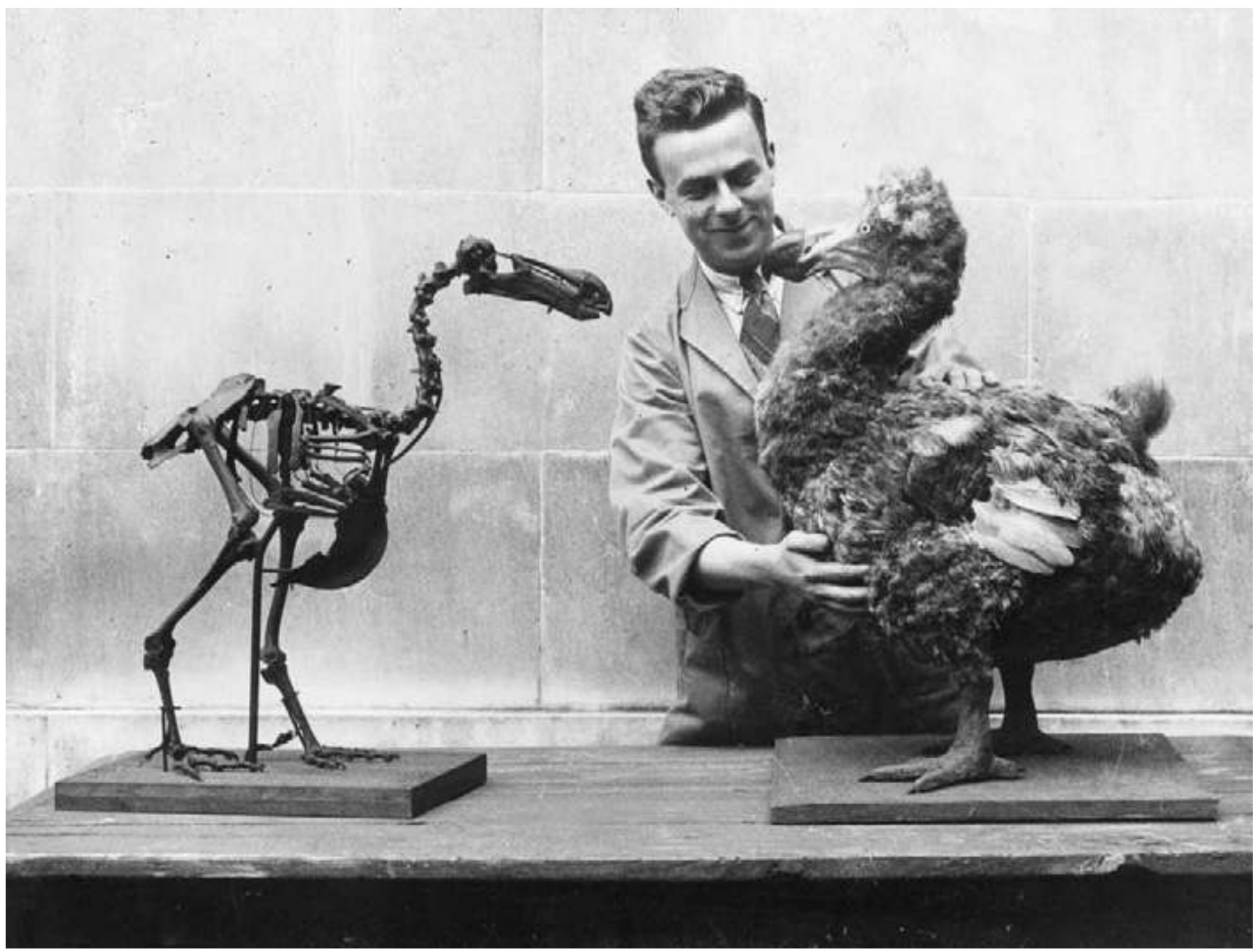

fig. 4

Squelette de dodo, 1938.

(c) Becker/Fox Photos/Getty

Images. 


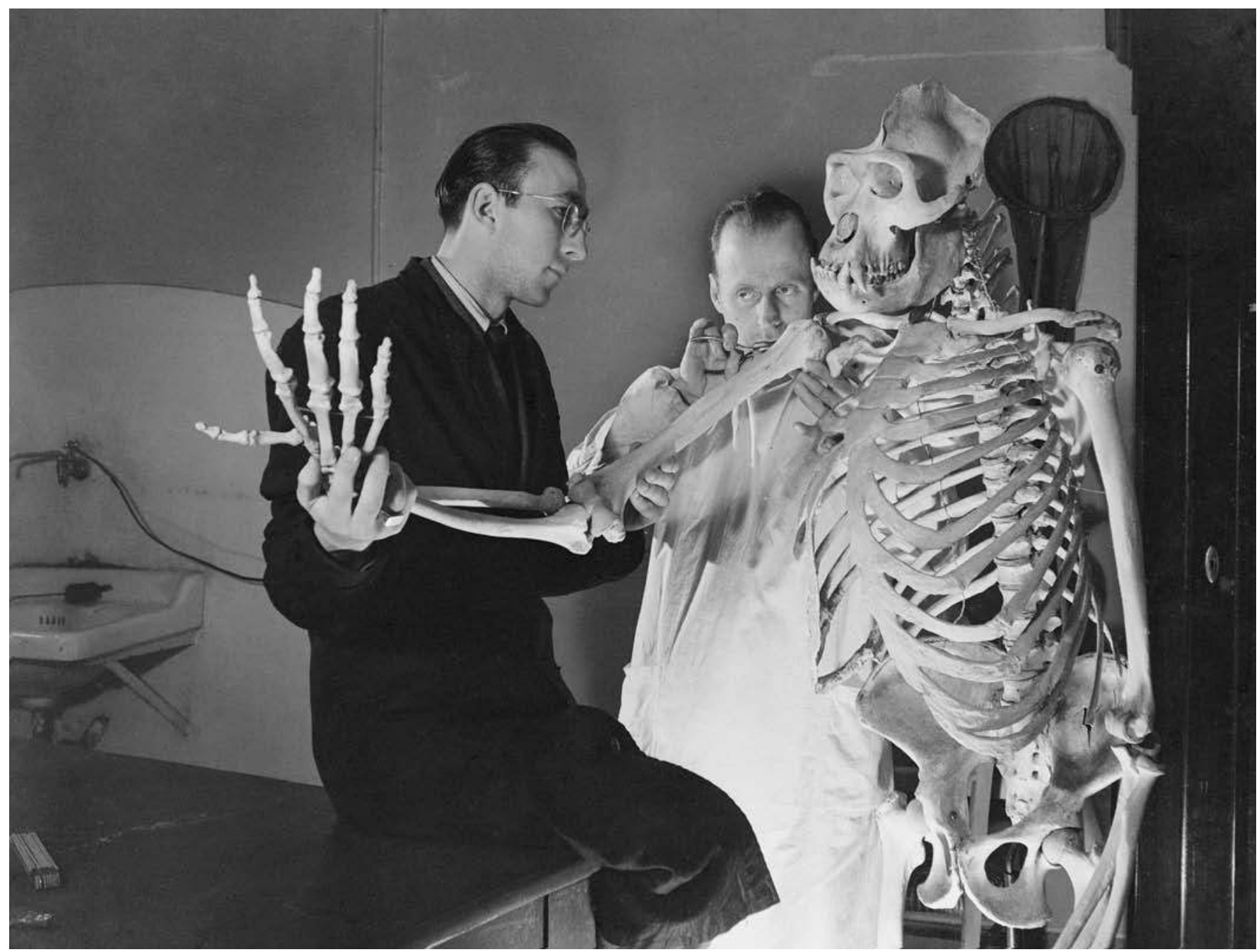

fig. 5

Squelette de gorille

au musée d'histoire naturelle,

Italie, vers 1955

(C) Archivio Cameraphoto

Epoche/Getty Images. 
milieu d'une rue et demande aux fouilleurs de faire attention. «Voyez cette route, elle est constamment refaite. On y a mêlé de la pierre, du goudron. Mais vous allez retrouver le même concept à l'intérieur des maisons. On ne cesse de faire et de refaire les sols constamment, avec de la bouse de vache, de l'eau... Les sols sont renforcés et, quand vous les fouillez, ce sont des couches parfois très épaisses et très dures parce qu'ils ont été refaits en permanence. Ce que vous découvrez quand vous mettez au jour de telles choses, c'est un état, un moment dans un processus de réélaboration continu qui s'est arrêté. » Le Dr P. continue sa marche. II y a de très belles maisons partout, mais il ne s'arrête pas, son regard est attiré par un grand trou qui sert de poubelle où des poules viennent picorer. Une chèvre pousse le derrière du Dr P., faisant rire les enfants rassemblés autour de lui, mais cela ne semble pas déranger notre archéologue qui poursuit son chemin. «Regardez maintenant vers le haut, cette petite construction à l'étage. C'est à la fois un deuxième étage et un toit, une sorte de balcon. Vous trouverez beaucoup de constructions de ce type, difficiles à classer. On ne sait pas si c'est un étage, un toit ou autre chose, cela ressemble à un étage ouvert transformé en toit, mais ce pourrait tout aussi bien être un toit transformé en étage. Et voyez aussi cet autre bâtiment. Le deuxième étage semble en cours de construction, comme s'il n'avait jamais été achevé. On a l'impression qu'il a subi une forme d'incision, que c'était un étage fermé qu'on a ouvert pour le rendre semi-public. " Comme la route et les sols des maisons constamment refaits, les étages du village constituent un work in progress.

Le Dr P. souligne l'inachèvement de l'espace, son regard (et du même coup le nôtre) est attiré par les signes de cette reprise constante des structures. «Cela paraît chaotique comme ça, ajoute-t-il, mais c'est la vie. Elle est faite de tas de petites constructions provisoires, fragiles, imparfaites. Avec votre truelle, vous passez souvent à travers tout cela, il faudrait développer un autre sens, une autre sensibilité, c'est la raison pour laquelle je vous montre tout ça. C'est déjà compliqué de les percevoir quand vous traversez un village habité, votre regard est attiré vers ce qui est solide, bien identifiable, structuré, ce qui saute aux yeux, c'est encore plus délicat quand vous êtes dans un trou. Regardez ce toit, il est fait d'un mélange de pierres et de briques posées les unes sur les autres et de tiges de bambou ficelées. On pourrait très bien imaginer un pareil tas au sol, mais pourtant c'est un toit. Ainsi, avec votre truelle, vous faites face à un tas, vous le traversez et vous vous retrouvez dans une maison, comme par enchantement, sans savoir que c'était un toit. "

«Faire face à un tas, le traverser... » est une expérience à la fois radicalement distincte des précédentes et en même temps similaire par son effet de contorsion. Acteur d'un film brouillé, le fouilleur est invité à deviner «le toit derrière le tas et à s'identifier à des personnages (le villageois d'à côté) qui pourraient avoir existé en cet endroit précis. Grâce à l'exercice d'immersion «ethnoarchéologique» proposé par le Dr P., notre image du village se modifie et se peuple de choses bien fragiles, inachevées, en cours de reconfiguration, une sorte de vaste chantier à ciel ouvert, un village en projet qui ne cesse de se recomposer en se dotant de nouvelles couches, d'extensions. Maisons abandonnées, en construction, routes rafistolées, amoncellements en tout genre: dans la déambulation de l'archéologue, 


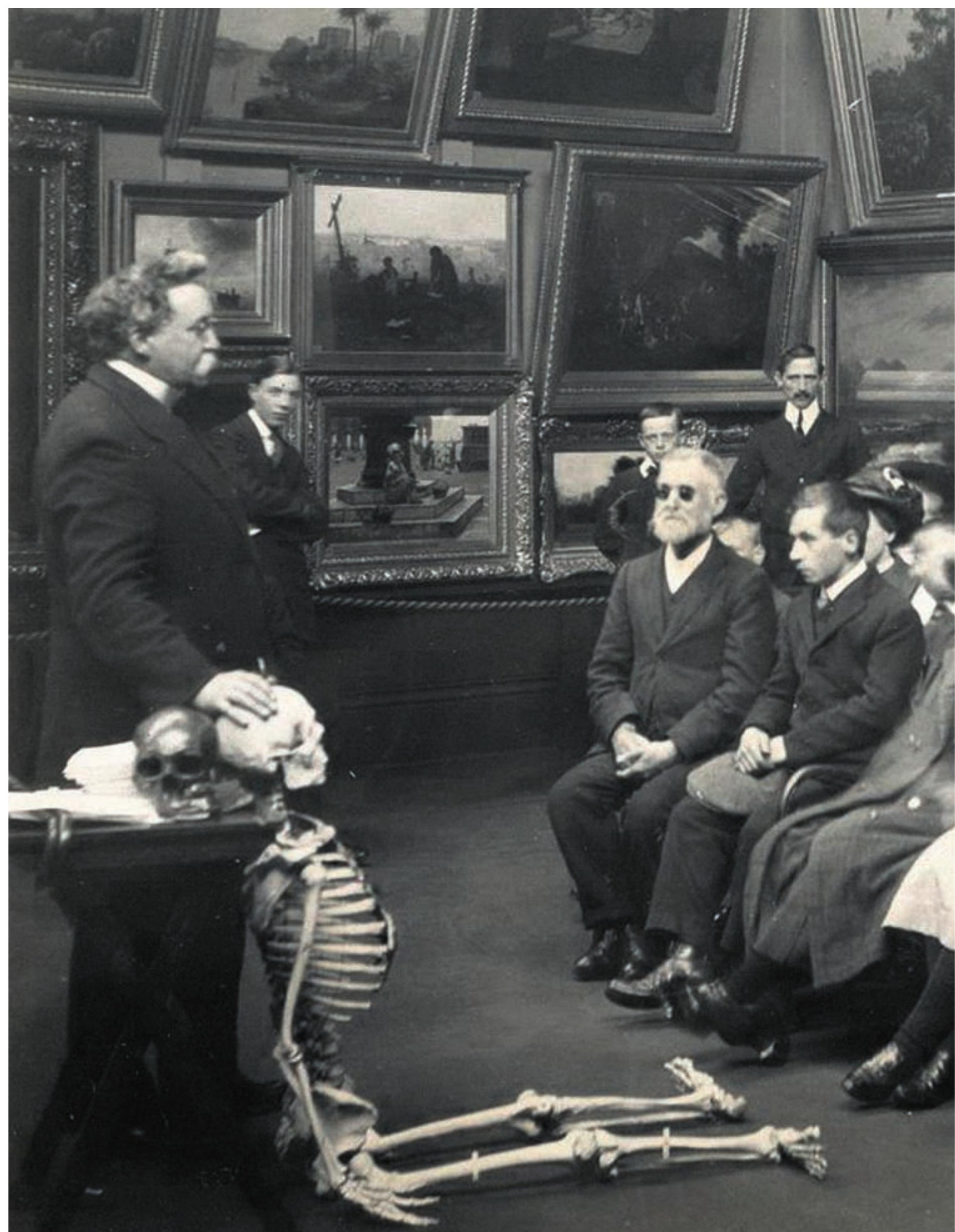


14. Le carré (ou le cube Iorsqu'on va en profondeur) est devenu l'unité conventionnelle du plan de travail archéologique durant la période coloniale (Wheeler 1954 ; Chadha 2002). Transformant les fouilles en gigantesques plans quadrillés, la méthode implantée par Sir Mortimer Wheeler lorsqu'il était à la tête de l'Archaeological Survey of India (ASI) est sans doute l'un des plus grands programmes de rationalisation des méthodes de fouille jamais réalisé. Elle est encore suivie en Inde aujourd'hui. le village physique opère sa mue, il devient un village de travail, une sorte de village «analogique». Notons que le Dr P. n'a affirmé à aucun moment que ce village était l'image vivante et animée de ce que nous devions trouver dans le sol, «en négatif». Son objectif était plutôt d'ouvrir la sensibilité de ses fouilleurs à d'autres possibilités, à des logiques de construction ou des agencements qu'ils n'auraient pas soupçonnés s'ils ne les avaient vus «de leurs propres yeux». Dans l'espoir que la civilisation qu'il exhume rejaillisse dans toute sa singularité, l'archéologue n'a pas d'autre choix que de travailler le répertoire des formes connues de ses fouilleurs, leur apprendre à suspecter des présences derrière les choses et les préparer à voir surgir de tout et si possible du plausible. Ainsi le village voisin est-il devenu leur village mental: un espace physique où déambulent des personnages en chair et en os et un espace virtuel dont ils sont les acteurs et dans lequel ils sont invités à simuler des scénarios.

\section{"Tranchée 301, kiln":}

\section{immersion dans un espace non identifié}

Attardons-nous maintenant sur la manière dont une tranchée change d'identité au fur et à mesure qu'elle se stratifie, générant plusieurs jeux de chaises musicales. En Inde, il est rare que les archéologues fouillent eux-mêmes, ils délèguent la supervision des carrés de fouille ${ }^{14}$ aux trench supervisors, et viennent régulièrement visiter les tranchées et donner leurs instructions. Voilà qui donne à la fouille une tournure interactive savoureuse. Ces visites sont parfois très denses en échanges intellectuels. Plus les gens expérimentés viennent le visiter, plus le fouilleur est susceptible d'entendre des hypothèses différentes. II doit dans tous les cas prendre des décisions pour avancer. Observons ces enchainements décisionnels de plus près. Le Dr P., le Dr S. et le chef du chantier emmènent les fouilleurs faire le tour du site. Ils s'arrêtent sur la seconde colline et remarquent un indice au sol: une série de pierres remarquablement disposées. Dans un premier temps, le $\operatorname{Dr} \mathrm{P}$. et le $\mathrm{Dr} \mathrm{S}$. s'accordent à dire que la rangée de pierres en surface est le signe qu'il pourrait bien y avoir un portail ou quelque chose comme la porte d'entrée d'une citadelle. L'existence d'un mur de fortification autour du site serait une découverte unique.

Prenons le même endroit au second jour. Deux tranchées ont été ouvertes côte à côte, auxquelles on a assigné un numéro: 301 et 302. Les fouilleurs décident de commencer également le travail dans un troisième carré adjacent (numéroté 310 ) et de progresser horizontalement. Le Dr P. veut qu'ils avancent au même niveau, simultanément, pour aboutir à un ou deux événements concomitants. II s'assied au bord de la tranchée et guide la truelle du fouilleur. "Va toujours du connu vers l'inconnu, de ce qui est déjà défini vers ce qui ne l'est pas ", répète-t-il. Quelques briques de plâtre sont apparues en 301 et par-dessus un mur de briques crues. Dans les interstices, de la cendre jaillit en grande quantité. Un peu plus tard, le Dr S. arrive à son tour. Plusieurs briques rouges et du charbon apparaissent en 310 , toujours au premier niveau. On n'est pas encore très profond. L'archéologue dit que le quadrant nord-est est moins intéressant que le quadrant nord-ouest. Changement de tactique. Selon lui, les pierres en surface datent de la période historique ou médiévale. On a atteint le deuxième niveau d'occupation, mais pas encore le plus important selon lui: 
le chalcolithique. En gros, on peut y aller à la pioche: «Pas la peine de gratter au pinceau, on n'y sera jamais! » dit-il.

Quelques heures plus tard, visite d'une autre fouilleuse sur les bords de la 310. Elle s'assied sur une chaise, contemple longuement les indices au sol et s'exclame: “Cette tranchée, c'est un vrai volcan! D'après elle, les pierres en surface pourraient bien être chalcolithiques et l'usage de la pierre au lieu de briques en terre crue indiquerait qu'il s'agit d'une plateforme rituelle. Troisième jour, au petit matin, visite du chef du chantier, diplômé en géologie. Après un moment de réflexion, il dit: «Maison écroulée? Hmm, c'est un incendie! » Puis il s'en va. Plus tard, le Dr P. s'installe à nouveau sur le bord de la 301 et y reste toute la journée car une étrange matière vient de faire son apparition, qu'il appelle «matière vitrifiée». II affirme qu'il n'a jamais rien vu de semblable en cinquante ans de carrière et qu'il faut l'envoyer immédiatement au laboratoire. Plusieurs fois, le Dr P. se met en colère car il n'y voit rien et demande au fouilleur de nettoyer au pinceau. Mais la matière friable n'en finit pas de sortir. «Brushing is a science!» dit-il. Le fouilleur se plaint: «Quand je brosse, il me dit de creuser et quand je creuse, il me dit de brosser!»

Le cinquième jour, le $\operatorname{Dr} P$. fait l'hypothèse que les briques brûlées exposées pourraient être les restes d'un four domestique. II confirme qu'il s'agit d'un four (chulaha) aux multiples plateformes mais les ouvriers sont sceptiques, ainsi que le chef du chantier. Ils suspectent le fouilleur de l'avoir créé avec sa truelle. Nouvelle visite du Dr P.: on a atteint le troisième niveau d'occupation. II dit au fouilleur que sa tranchée est pleine de «choses s'apparentant à un mur». Selon lui, il faut tracer le mur révélé en 301, le reste étant sans intérêt, en particulier l'hypothèse du four. II a suffi de creuser un peu pour que celui-ci perde sa forme. «Ce ne sont que des briques brûlées. Pour parler de four, il faut une forme bien définie! » En 301, une rangée alternant briques et pierres a été exposée. Les fouilleurs restent toute la journée sur le bord du carré, cherchant une explication. Puis le Dr P. s'en va. Un archéologue de l'Archaeological Survey of India (ASI) arrive de Delhi et critique la méthode suivie jusqu'ici pour exposer la matière vitrifiée. Selon lui, il faut faire une coupe interne (une section): "L'archéologue doit chercher des images claires, mais là tout est confus! » s'exclame-t-il.

Après le départ du Dr P., le chef du chantier s'impatiente. II prend à part le fouilleur de la 310 et lui dit qu'il faut être plus radical et creuser plus profond. «II ne faut pas suivre la méthode américaine ici, c'est improductif, fouillons à la manière locale! » II prend la truelle et commence à gratter luimême avec détermination. On réalise vite que le mur n'est pas continu entre la 301 et la 310 , c'est un «mur fantôme». La matière compacte exposée en 310 est identifiée comme étant de la bouse de vache et il y a là, d'après le chef du chantier, une série de sols d'habitation: "Un effort considérable a été nécessaire pour faire de tels sols. Regardez comment les villageois refont le sol de leurs maisons. Imaginez cela après plusieurs siècles! »

Le septième jour, le Dr P. revient (toujours avec la même chaise pliante) et, regardant les traits de la 310, me confie: «J'aime cette tranchée parce qu'elle est simple. Dans le quart sud-ouest, c'est simplement du déchet. Regarde ces briques. Elles ont dû tomber d'une maison ou ont été jetées 

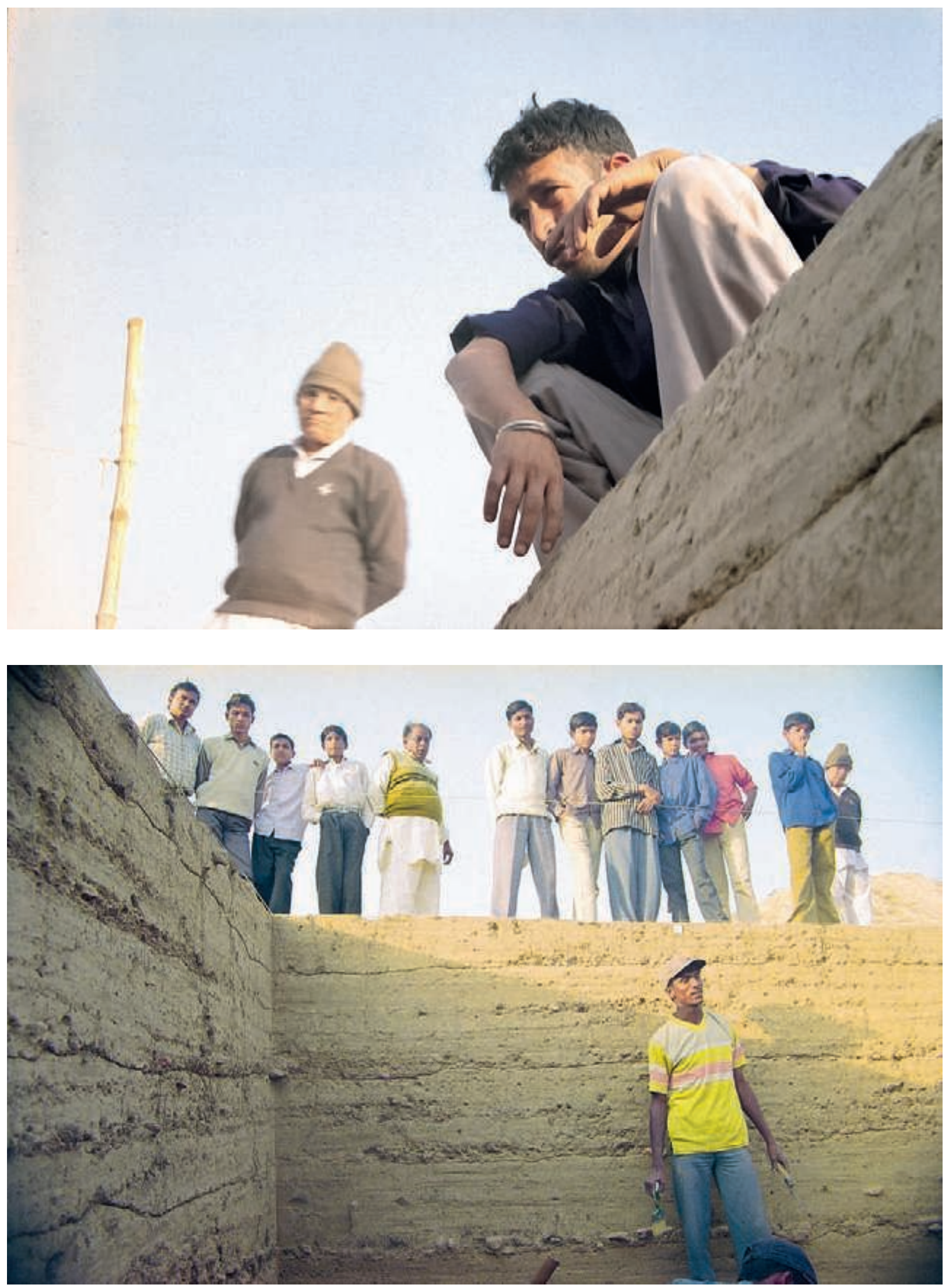
par les gens. C'est comme un trou où ils jetaient ce qu'ils n'utilisaient pas. » Un peu plus tard, on tombe sur du charbon, de la cendre et des briques brûlées en grande quantité donnant à la tranchée un visage apocalyptique. Le fouilleur attend les instructions. Le Dr P. dit: «Quand je fouille, j'aime m'entourer d'hypothèses et osciller entre elles. Il devrait en être de même pour vous! » Puis il disparaît. Quelques heures plus tard, on atteint la couche labélisée «numéro 4 ». Selon le chef du chantier, le petit mur exposé pourrait avoir été la base d'un pilier. «Quelque chose d'important, peut-être un rituel...» Puis, jetant un coup d'œil dans la 310, il confirme l'hypothèse précédente de l'incendie: «Des maisons brûlées, des sols calcinés, l'heure est grave!»

Le huitième jour, nouvelle visite du Dr P. Pour lui, la poubelle est la meilleure hypothèse de travail en 310: «Enlevez toutes ces briques brûlées, vous trouverez un mur de fondation, sinon c'est une poubelle. » Et il ajoute: "J'imagine toujours, en me promenant dans le village, ce qu'on en retrouverait s'il était recouvert de sable. Sans doute de nombreux endroits comme celui-ci, où tout est mélangé, et beaucoup de poubelles. »Plus tard, visite du chef du chantier, qui semble en mesure de proposer une séquence. Pour lui, le fouilleur doit noter trois éléments importants. «Premièrement, ces gens ont fait des sols très élaborés. Deuxièmement, ils ont creusé un trou pour y jeter des choses puis ce trou a été utilisé et réutilisé. Enfin, ils ont jeté des pierres autour des arbres, c'est pourquoi vous trouvez tant de pierres ici. » "Ce que vous fouillez, ajoute-t-il, c'est la façon dont les gens creusent, font des sols, des trous, jettent des pierres et organisent leur poubelle. L'archéologie, ce n'est que du bon sens et de l'observation. Mais plus vous aurez d'imagination, plus vous serez un bon archéologue! » Un peu plus tard, des visiteurs, des enfants des écoles et des villages alentour, s'amassent autour de la tranchée. La nouvelle a circulé qu'une fouille avait lieu. Le fouilleur en 310 explique: «Ceci est une poubelle et là, vous avez un mur. Une maison a dû s'écrouler et ces pierres en surface sont les restes d'un lieu rituel. »

Neuvième jour, visite du Dr $\mathrm{S}$.: on a trouvé une pièce moghole à un niveau chalcolithique. La découverte est inquiétante. On demande à un ouvrier musulman parlant ourdou de lire la date écrite sur la pièce de monnaie: 1408. Cette pièce cache une présence impromptue. Le chef du chantier se demande en effet si la colline n'aurait pas été remuée par une fouille précédente. Un archéologue indien célèbre a fouillé ici dans les années 1950 et a été contraint pour des raisons administratives de quitter le site sans laisser de rapport. II a ouvert plusieurs tranchées, essentiellement dans la partie appelée «le grenier» (granary area). Ce fait était connu mais on se demande s'il n'a pas aussi fouillé dans la partie sud du site, aire de l'actuel chantier. Le chef du chantier montre au fouilleur la partie dont il est sûr qu'elle a été fouillée par l'archéologue précédent, à cinquante mètres de la 310, derrière les buissons. "Nous avons aussi trouvé des sacs en plastique et des étiquettes en métal utilisées par l'ASI ainsi qu'un vieux stylo dans une autre tranchée», ajoute un fouilleur. D'après lui, le grand trou visible en 310 pourrait être le résultat d'une tranchée ouverte par l'équipe antérieure. Visite conjointe du Dr P. et du chef du chantier: d'après le premier, l'hypothèse du second n'est pas convaincante car, quel qu'ait été le travail mené par l'archéologue dans les années 1950, il s'est arrêté avant d'atteindre le niveau intéressant. «Pourquoi donc une telle hypothèse? » demande-t-il. Le chef du chantier répond: «Pourquoi pas? »

\section{ci-contre}

fig. $7 \mathrm{a}$ et $\mathrm{b}$

Au bord d'une tranchée archéologique sur une fouille en Inde, Rajasthan.

(c) Emmanuel Grimaud. 


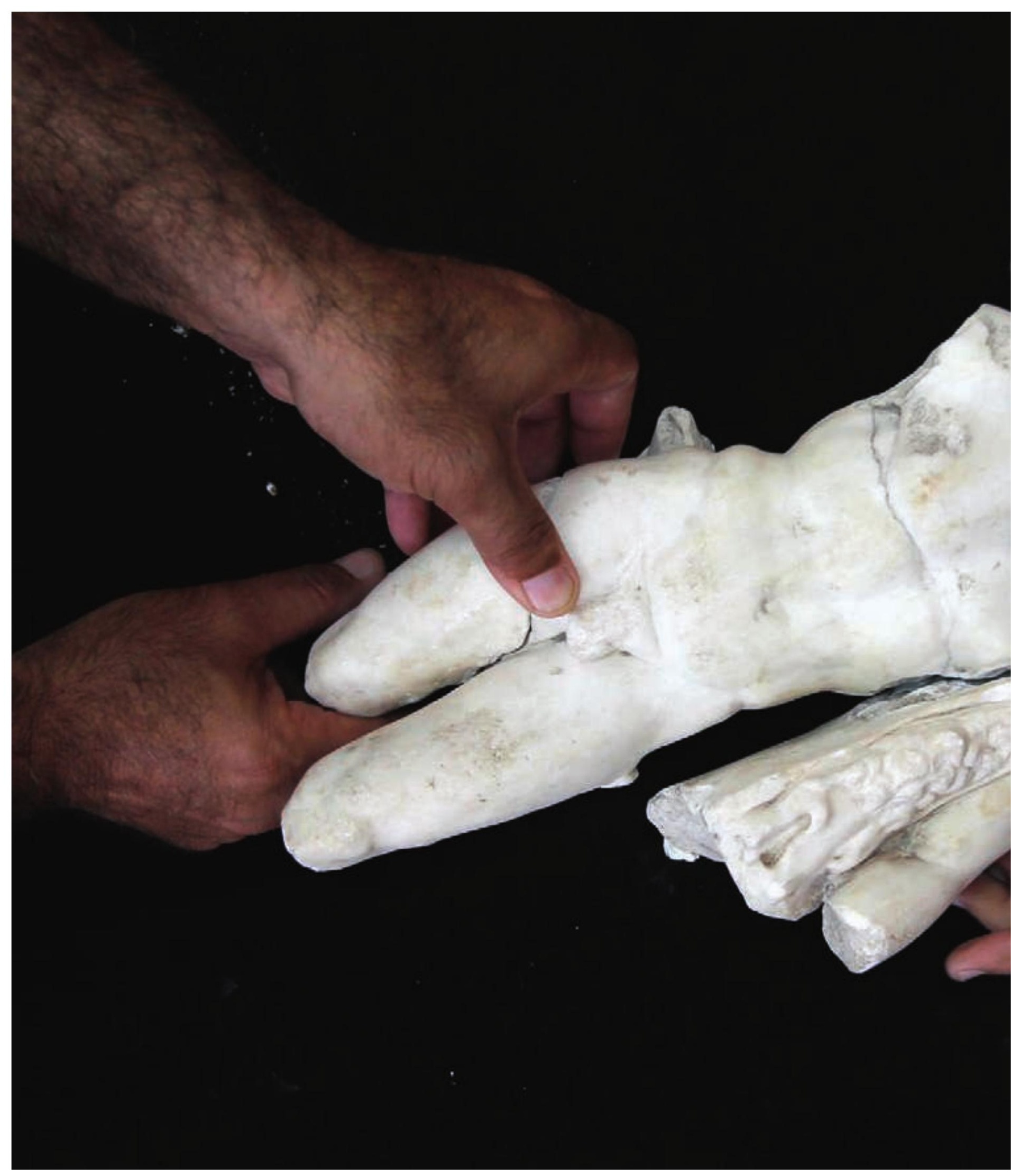


Dixième jour, nouvelle visite du Dr S.: les traces d'un mur apparaissent près de la matière vitrifiée toujours non identifiable. L'archéologue pense qu'il pourrait bien s'agir de la porte de citadelle que l'on cherche. Onzième jour: selon le Dr P., la tranchée se caractérise principalement par la présence de cette matière vitrifiée. II n'a jamais vu un aussi grand kiln de toute sa carrière. Le mot a été prononcé: c'est un kiln (une sorte de four) et la colline pourrait bien être une zone industrielle unique pour cette période. Mais l'assistante de l'archéologue indien intervient: elle pense qu'il s'agit d'une poubelle et non d'un four car le sol au-dessous n'est pas du tout calciné. Pour elle, la matière vitrifiée a été déposée ici, mais vient d'ailleurs. Quant au chef du chantier, il estime que ce n'est ni une poubelle ni un four mais qu'il faut tendre vers autre chose. II se garde cependant de faire toute nouvelle hypothèse. Douzième jour, le Dr P. revient seul. Pour lui, la séquence est maintenant clairement visible. II appelle tous les fouilleurs et leur demande de la noter: «En 301, en allant à reculons, du plus contemporain au plus ancien, on a du remblai (1), un mur sans briques (2). [II marche sur la tranchée et pointe chacun des éléments.] Ce mur recouvre un autre mur orienté mi-ouest (3). De grandes briques (4) recouvrent un kiln en 301 tout d'abord constitué du déchet du kiln (5). Ce déchet repose sur le sol du kiln (6) et est séparé du sol par cinq à dix couches de cette matière épaisse, blanche et cendreuse, avec quelques taches de noir (7). Vers le sud, une rangée de briques crues (8) repose sur un mur de pierres (9) qui court de la tranchée 302 vers la 301. Les pierres de ce mur étaient visibles avant la fouille. En 302, le mur en pierre pourrait bien reposer sur un mur de briques (10). Je voudrais que vous me confirmiez le "pourrait bien". C'est une information importante. Si mon hypothèse est correcte, en allant du plus ancien vers le plus contemporain, c'est une image très claire. » Le Dr P. pense qu'il faut laisser la tranchée en l'état car on n'obtiendra rien en allant plus profond.

D'autres changements eurent lieu ensuite, la séquence fut étendue, précisée, à nouveau débattue. Si l'ordre de cette dernière ne faisait pas l'unanimité, tous s'accordaient à dire qu'il s'agissait d'une «séquence remarquable», un «matériau unique», que cette colline montrait des «traits exemplaires», une «stratigraphie extrêmement précise » et que cela pourrait bien être «une zone industrielle». Quand le Dr S. installa son échafaudage pour la photographie, on demanda aux ouvriers de bien nettoyer la poussière et sur l'écriteau noir qui ressemblait à un clap de cinéma, disposé dans le champ de la photo, l'archéologue marqua à la craie: «Tranchée 301, kiln ».

J'ai délibérément décrit l'épisode dans sa totalité, malgré sa longueur, pour que l'on voie comment se sont succédé ici avec une intensité rare les interrogations et les affirmations, les actes de regard et les prises de position. Revenons à l'expérience de Heider et Simmel, qui distinguait au moins deux types de publics et différents types d'attribution, plus ou moins libres. II y avait d'abord, dans le public, ceux qui collaient aux formes et appelaient les choses par leur nom: un triangle est un triangle, un carré est un carré (les archéologues prudents); puis ceux qui anthropomorphisaient par commodité et appelaient le grand triangle «papa», le petit «bébé» et le rond «maman » (les fouilleurs qui n'hésitaient pas ici à parler «rituel», «événement grave», «citadelle», «zone industrielle»). Entre les deux, 
certains bricolaient et parlaient de «papa Triangle» et de «maman Triangle» (le fouilleur pris en étau entre les indications des archéologues prudents et les autres). Et il y avait surtout une grande variété de scénarios différents selon les points d'accroche choisis (le mouvement ou l'action d'abord, telle ou telle figure ensuite, ou bien telle figure d'abord puis l'action ensuite). On ne peut s'empêcher ici de rechercher des causes au mouvement (qui fait quoi, qui déclenche quoi, qui chasse l'autre ou le repousse). Sur la fouille, où les traces sont immobiles, le déroulement des actions n'est pas donné d'emblée, il est toujours inféré. II serait simpliste de réduire ces jeux de piste à des variations de points de vue comme si tout était dans la tête du fouilleur. Comme dans l'expérience de Heider et Simmel, le paysage perçu ne cesse de se modifier. Avant les points de vue, c'est la tranchée qui change d'état, obligeant le fouilleur à adapter son corps d'hypothèses.

Ainsi les indices se redistribuent, contraignant les fouilleurs à poser leur truelle pour se mettre à la place de ces présences virtuelles dont ils exhument les restes avant de creuser de plus belle. Et pour stimuler de nouvelles hypothèses, ils ne cessent de se remettre en position de spectateur, en «contemplation active", dans leur propre carré de fouille comme dans celui des autres, s'asseyant sur le bord pour en déchiffrer les indices à distance. L'intensité des changements de position varie en fonction de la difficulté à identifier ce dont il s'agit. Si les possibilités de cours d'action en ces lieux sont infinies, plus l'identité des objets est incertaine, moins ils sont "parlants», plus les tentatives pour en forcer l'expression se multiplient. Des options divergentes se font entendre, renvoyant chacun des camps à la pauvreté du vocabulaire pour qualifier "l'inédit» de ce à quoi ils ont affaire. Certes, les possibilités de lieux n'étaient pas légion, mais suffisantes pour que de multiples hypothèses s'expriment, que des arguments parfois virulents soient échangés. Si l'entité «four» trouva ses défenseurs au même titre que l'entité «poubelle» eut ses porte-parole (ainsi que la «zone industrielle», la «porte de citadelle», la «maison d'artisan» et la «plateforme rituelle»), le jeu des prises de parole était étroitement dépendant des fluctuations mêmes du paysage d'indices de la tranchée 301. Dans cet espace au départ non identifié, on n'a pas oscillé une fois seulement entre la matière et des hypothèses projetées, «imaginaires». À l'intérieur de cette zone de présences potentielles, toujours «en surplus » par rapport aux mots, un scénario s'est élaboré tant bien que mal, après un nombre incalculable de repositionnements du regard, d'oscillations entre plusieurs entités plausibles et d'ajustements entre les mots et les choses.

\section{Quand tout le monde s'en mêle: énigme et interactivité}

Ainsi la fouille offrirait des tas de petites expériences de représentation de différents ordres: des expériences immersives qui obligent à franchir les dimensions (du plan à l'espace stratigraphique), des expériences d'animation à partir d'indices dévitalisés qu'il faut ramener à du vivant, des expériences limites d'attribution à partir de faisceaux d'indices. Grâce à elles, les plausibles se confirment ou s'infirment. Lorsque le public s'en mêle, le bord des tranchées devient une arène d'expression encore plus interactive. L'effort de représentation se redistribue. Le jeu des chaises musicales intègre de nouveaux acteurs et spectateurs et, avec eux, de nouvelles possibilités de varier les positions, ne serait-ce que pour «voir ce qui se passe». 


\section{ci-contre}

fig. 9

João Maria Gusmão et Pedro

Paiva, Ventriloquie, 2009.

Avec l'aimable autorisation

des artistes et de Sies + Höke,

Düsseldorf.
Le site indien où nous nous trouvions était visité par des gens qui ne faisaient pas toujours partie de l'équipe des archéologues, ce qui ne les empêchait pas de participer à leurs boucles interprétatives. Sur le bord des tranchées se multipliaient les épreuves d'interprétation, proches de puzzles collectifs ou de jeux de questions-réponses. Inutile de dire qu'on frôlait souvent la cacophonie; il arrivait que l'archéologue impose le silence tant les gens s'amassaient tout autour pour assister au spectacle. La plupart du temps, les visiteurs essayaient d'identifier ce qui apparaissait et s'en allaient: "Un mur? Très bien, partons, allons voir ailleurs!» «Pas de trésor? Allons-nous-en!» Mais il arrivait aussi qu'ils s'installent pour plusieurs heures et engagent la conversation avec le fouilleur. II suffisait d'un trou, d'un point de jonction, d'une diagonale, d'une ligne, d'un angle ou encore de murs parallèles pour stimuler les conversations. Mais comment passe-t-on d'un jeu de devinettes sur des angles, des lignes et des courbes à une discussion d'ordre scénaristique ou même à des récits mettant en scène des personnages en chair et en os?

Les lignes sont faites pour être prolongées et les objets pour être complétés, surtout lorsque le site peine à s'actualiser comme monde. Un vieil homme qui visiblement avait une certaine notoriété dans son village s'approcha un jour des archéologues et leur dit: «Ici vous trouverez le squelette de Lord Thomas, le général de Napoléon. Vous trouverez aussi le Mahabharata et les Veda! " C'est moins la revendication d'une aptitude visionnaire qui compte dans cet exemple que l'expression d'une certaine attente que la fouille comblera ou non. En effet, si quelque chose d'intéressant devait être trouvé, quoi de plus naturel qu'un squelette, pourvu d'une identité et, si possible, celle d'un personnage connu qui nous raccroche à la grande histoire? II arrivait que s'asseyent aussi des gens qui prétendaient déjà tout savoir, chacun y allant de ses prophéties. Sachant que d'autres archéologues présents dans la région cherchaient la rivière Sarasvatî (un repère essentiel de la mythologie indienne), un homme dit: «lci, vous trouverez le point de jonction entre nos deux rivières sacrées! » Nul besoin de statues, d'antiquités ou de scénarios spectaculaires pour que l'imagination soit stimulée. Des configurations géométriques simples (un point de jonction) généraient déjà une émulation. Dès que l'on trouvait quelque chose ressemblant à un objet, les spectateurs cherchaient à recomposer la partie manquante, s'appuyant sur ce que les spécialistes de la perception visuelle appellent la complétion modale, cette tendance du système perceptif à compléter les images inachevées. C'était souvent à plusieurs que se faisait cette reconstitution de formes humaines ou animales. On essayait de les rapprocher de silhouettes connues, d'imaginer les fonctions ou les usages des objets. On se rapprochait volontiers pour toucher et deviner, sachant que beaucoup d'objets étaient en morceaux et qu'il n'était pas toujours facile d'y voir des formes identifiables. Nombreux étaient ceux qui attendaient des heures que la partie manquante d'un objet soit retrouvée, comme si la fouille produisait, au fur et à mesure de sa progression, des configurations de stimuli pour des puzzles à l'infini. Et lorsque les couches commençaient à s'imbriquer les unes dans les autres, que le paysage de la tranchée se complexifiait, c'était l'occasion de s'exercer à une autre forme de curiosité, stratigraphique cette fois: on cherchait à deviner quelle structure était préalable, quel mur avait été construit en premier, quelles briques avaient 


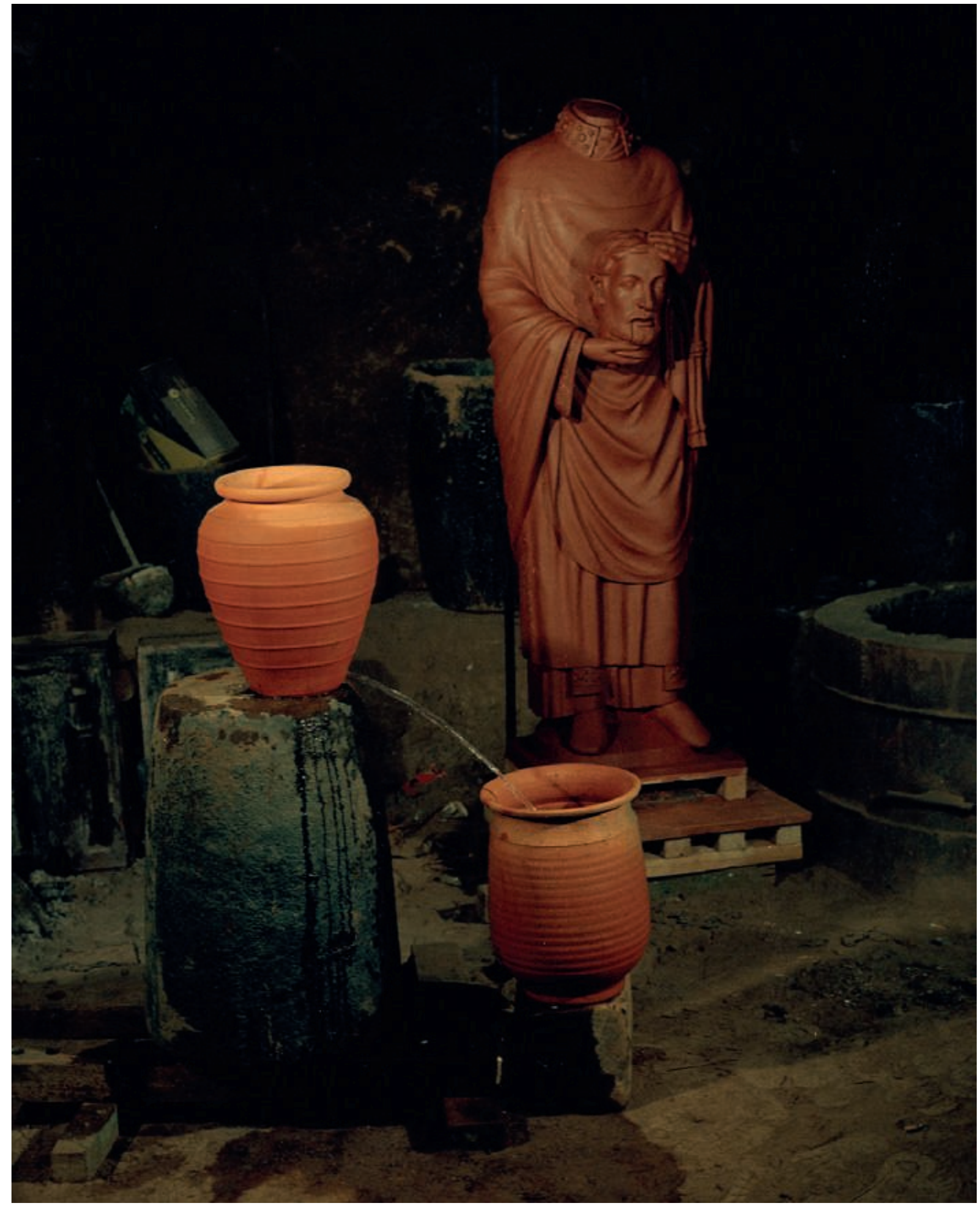




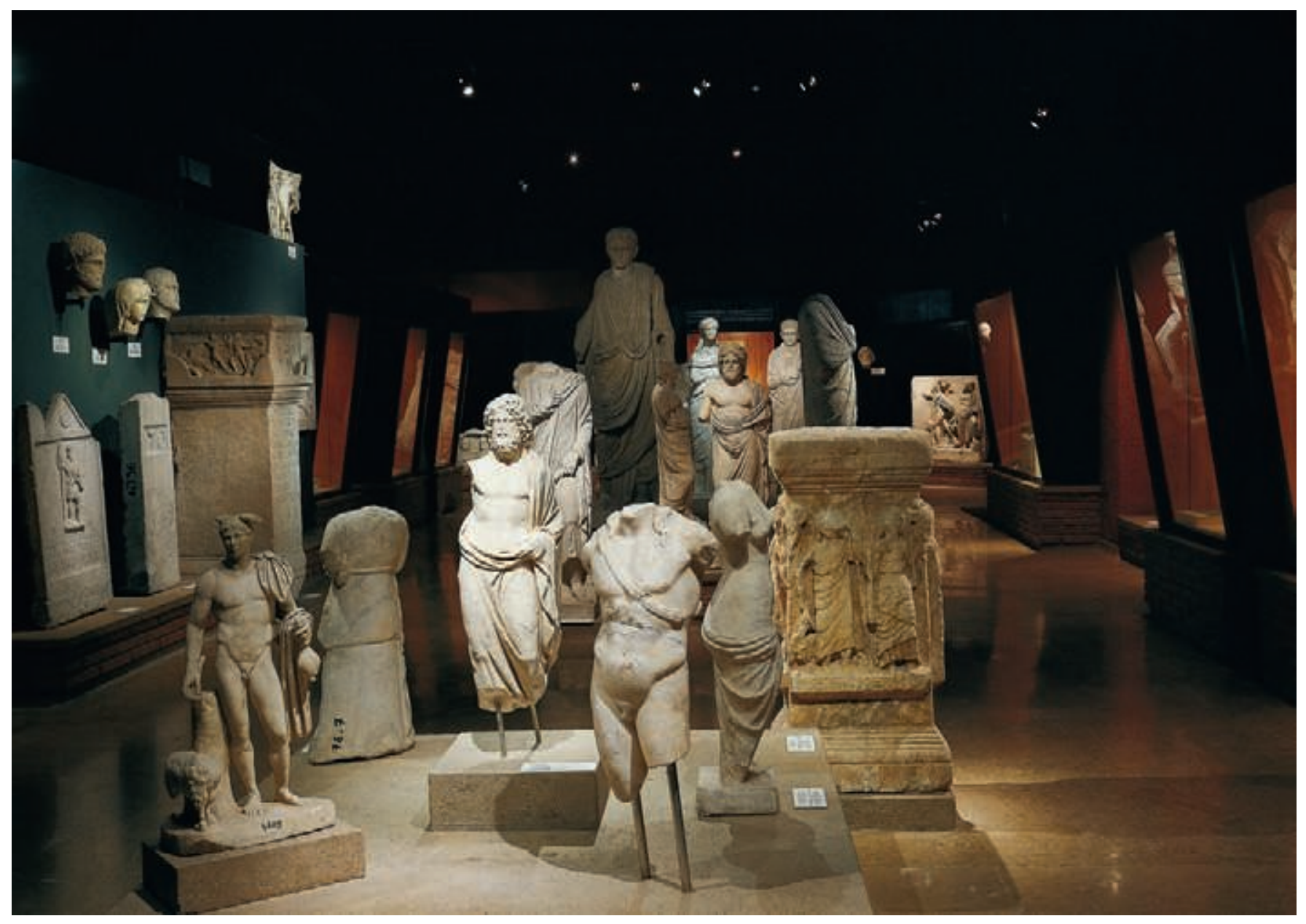

fig. 10

Salle des sculptures romaines d'Asie mineure. Musée

d'archéologie d'Istanbul,

2007. () DeAgostini/Getty

Images. 
été réutilisées et dans quel but. Le fouilleur devait en permanence faire face au désir de l'audience de voir des formes identifiables et plus complètes. Ces «visites » devenaient pour le public l'occasion d'exercer sa perspicacité et pour le fouilleur de l'attiser.

Tentons de mieux saisir ce qui se joue dans cette multiplication des expériences indicielles. La fouille est bien plus que la scène d'un spectacle où les gens viendraient contempler l'émergence d'un passé enfoui: elle devient le lieu d'un jeu d'immersion collectivement partagé. Tandis que les fouilleurs sont «dedans», les visiteurs qui s'asseyent sur le bord et contemplent le processus du «dehors» participent à l'exercice de recomposition sur le mode des images ambiguës. Le bord de la tranchée agit comme une caisse de résonance pour des illusions d'optique en tout genre. Il génère par exemple à la pelle des «figures fictives» (au sens de Gaetano Kanisza ${ }^{15}$ ). Il est le lieu de toutes les équivoques, de tous les délires interprétatifs et confusions possibles ${ }^{\mathbf{1 6}}$. Depuis l'intérieur de la tranchée, certaines connexions sont jugées viables, d'autres non, et quand elles ne sont pas corroborées par les données, les archéologues finissent par les écarter comme des figures impossibles. Là où l'archéologue voit ces différences, le visiteur lui, a tendance à «niveler» ces paysages d'indices et à discerner une continuité là où il n'y en a pas. Entre archéologues et visiteurs, les malentendus sont classiques. Mais du point de vue qui nous intéresse ici, à savoir celui de la propagation de l'expérience indicielle, le malentendu est fertile, sans altérer fondamentalement la nature de l'expérience. Plus une fouille génère de paysages d'indices complexes et différenciés, et plus il y a de gens pour les interpréter, plus le bord des tranchées devient le lieu d'un rapport de forces entre des expériences de représentation différentes qui tantôt s'enrichissent de nouveaux éléments, tantôt s'appauvrissent, selon les experts en présence. Une énigme en cache une autre. Une expérience indicielle aussi, dans la mesure où l'impossibilité fondamentale de reconstituer se voit remise en jeu à chaque fois que l'archéologue avoue ne pas pouvoir aller plus loin dans l'interprétation.

Les questions du public constituent une drôle d'épreuve pour les archéologues. Elles les obligent à s'expliquer et à parler au nom des choses qu'ils exhument. Comment en effet ne pas être étonné par cette faculté du fouilleur expérimenté qui lui permet de constituer du récit de manière souvent impressionnante à partir des maigres données dont il dispose? On l'aura compris, il n'y a rien de mieux pour stimuler les esprits et même les échauffer qu'une fouille en train de se faire qui multiplie les contorsions perceptives autour de faisceaux d'indices non stabilisés. On peut s'interroger, dans ce contexte, sur le statut à accorder à ces petites phrases ironiques prononcées par le public et qui visent la plupart du temps à s'approprier les objets euxmêmes ou à les détourner au profit de scénarios absurdes. Serait-ce une manière de mettre à distance le processus de fouille et ses motivations? À moins que ce ne soit une façon pour le visiteur d'évaluer sur ce lieu précis, dans ce carré de fouille-ci, où se situe l'impossibilité de reconstituer car c'est cette difficulté qui détermine les règles du jeu des chaises variables et en conditionne les possibilités d'ouverture, permettant ou non la participation de nouveaux entrants (acteurs, spectateurs ou personnages). Ainsi, lorsqu'on découvrit une figurine de cheval en terre cuite cassée, un homme
15. "Le système optique comble toujours les lacunes, va toujours au-delà de l'information donnée, au moyen de l'interpolation perceptive ", écrit Gaetano Kanisza (1997 : 59-60).

16. Lorsque la mythologie s'invite dans le débat archéologique, c'est d'ailleurs souvent sous la forme de discussions autour d'illusions d'optique. Certains voient des choses là où d'autres ne voient rien. Un exemple remarquable est la controverse autour de la présence ou de l'absence du cheval védique à Harappa. Celle-ci a pris la forme d'un débat autour d'un sceau falsifié sur Photoshop (Witzel et Farmer 2000). 
s'approcha et dit: «La pauvre créature a la jambe cassée à force d'avoir escaladé la colline!» Un autre ajouta: «Il faut lui donner à boire!» Puis, faisant face à un squelette, il lança: «Regardez, le squelette nous sourit!» Un autre lui répondit: «Sa tête est penchée, il doit être en train de dormir!» Ce à quoi quelqu'un rétorqua: «Moi, je ne le touche pas, je suis végétarien!» L'objet qui constituait le stimulus du jeu de questions-réponses n'était plus pris ici dans des scénarios inspirés de la grande histoire ou de la mythologie, mais dans une multitude de petits scénarios, de petites histoires qui frôlaient l'animisme sur le mode de la plaisanterie. Si n'importe quel visiteur pouvait faire parler un objet et nouer avec lui un rapport ventriloque, la plupart des commentaires de ce genre s'appuyaient sur un handicap: une jambe cassée, un bras manquant, un nez amputé étaient prétextes à inventer des histoires incongrues. Tout le monde savait bien que rien de tout cela n'était vrai, que ces petits scénarios dérisoires n'étaient pas sérieux. Mais, sur le moment, ces «petites phrases» avaient pour vertu de contribuer au jeu d'interprétation en invoquant par défaut de l'improbable pour retrouver du plausible, d'animer des objets inertes ou bien de mettre à distance la prétention même à leur redonner vie.

\section{Conclusion}

Ainsi, le silence de la matière pousse ceux qui s'y confrontent à un repositionnement constant pour mieux voir et ces changements de position conditionnent largement les prises de parole. La tranchée archéologique aspire à devenir une "salle de projection", même s'il s'agit d'un drôle de film dont les personnages principaux sont des présences non identifiées. Les archéologues savent bien que le sol ne parle que par ellipses, mais ils modifient leurs scénarios en fonction de ce qu'ils trouvent, ils sont contraints de faire du cinéma d'animation sans le savoir ou une forme de cinéma sans caméra. Dans ce contexte, les jeux de chaises observés constituent des moments de mise en contact avec le passé sur un mode profondément irrégulier et discontinu. II s'agit d'expériences aux frontières de la ventriloquie à double sens: des appareils perceptifs s'emparent de choses pour les faire parler, et ces choses passées et muettes parlent à travers eux. Dans tous les cas, c'est l'impossibilité d'une immersion pleine et entière dans un espace vivant en trois dimensions qui crée du jeu, générant des expériences d'attribution, d'animation et d'interpolation sur un mode frénétique.

Plus les chaises se multiplient au bord de la tranchée, plus le film a de chances de s'éclaircir mais aussi de se brouiller. La quantité de ventriloquie autorisée dans cette arène d'expression (qui l'est autant pour des choses, des scénarios, que pour des hommes) semble indexée ici à une impossibilité de reconstitution foncièrement fluctuante, l'idéal pour les fouilleurs étant ce moment où les indices «parlent d'eux-mêmes». Rappelons-nous notre archéologue immergé au fond de son trou face à un squelette car il avait trouvé une solution pour assumer sans complexe ce renversement, lui donnant l'allure d'un véritable dispositif de contorsion oculaire (autant qu'oraculaire). Plutôt que de considérer l'archéologue comme un réalisateur à la poursuite d'un film impossible, il valait mieux pour lui s'assumer comme personnage (d'un film dont il n'avait pas les clés) ou bien comme spectateur (d'un film inversé), car ce sont surtout les choses elles-mêmes qui passent leur temps à faire leur cinéma et, avant les choses, les hommes du passé, 
qui se sont adonnés à toutes sortes d'actions qu'on ne peut, pour la plupart, retracer. Contenant une dramaturgie cachée, ces faisceaux d'actions diverses qu'il faut recomposer mettent le fouilleur en position de spectateur hyperactif de scénarios cryptés qui se déroulent à l'envers. Le cinéaste n'est pas ici celui qu'on croit. Les actions qui se sont produites il y a bien longtemps composent un cinéma d'animation solide qui se déroule à la fois à l'envers et en négatif (ou partiellement masqué ${ }^{17}$ ), surgi des profondeurs de la terre. Ce cinéma fragmentaire posséderait alors sa vitesse d'exécution propre (en indices/siècle plutôt qu'en images/seconde). Les traces en seraient les principaux acteurs, mais le scénario d'origine nous échappe. Si elle est peut-être très personnelle, cette théorie est néanmoins assez fidèle aux acrobaties de la perception exigées par l'expérience de fouille. Après s'être mis dans la peau du personnage amnésique de Memento, l'archéologue peut continuer à jouer au jeu des chaises (et des choses) et doper son imagination par de nouvelles expériences de représentation bien plus confortablement que dans une épistémologie qui n'assumerait pas, au sein de ces expériences elles-mêmes, ce que Carlo Ginzburg appelle l'« entrelacement du vrai, du faux et du fictif » (Ginzburg 2010 [1986]: 17).

CNRS-LESC emmanuel.grimaud@gmail.com
17. Notons que Heider et Simmel montrèrent leur film à l'envers dans la dernière phase de leur expérience pour voir dans quelle mesure l'attribution causale était dépendante de l'ordre des éléments et de la temporalité des actions et pas seulement des caractéristiques des figures. Mais ils n'allèrent pas jusqu'à le montrer en négatif ou en masquant des parties des figures. 


\section{Audouze, Françoise et Perlès, Catherine}

1980 "L'ethnoarchéologie ", Les Nouvelles de l'archéologie 4 : 7-10.

\section{Bergson, Henri}

1907 «Le mécanisme cinématographique de la pensée et l'illusion mécanistique ", in L'Évolution créatrice. Paris, PUF.

\section{Binford, Lewis}

1980 "Le rôle de l'ethnoarchéologie dans la recherche archéologique ", Les Nouvelles de l'archéologie 4: 31-33.

\section{Bryant, Edwin et Patton, Laurie (éd.)}

2005 The Indo-Aryan Controversy: Evidence and Inference in Indian History. Londres-New York, Routledge.

\section{Chadha, Ashis}

2002 "Visions of discipline. Sir Mortimer Wheeler and the archaeological method in India (1944-1948) ", Journal of Social Archaeology 2(3) : 378-401.

2010 «Cryptographic imagination: Indus script and the project of scientific decipherment ", Indian Economic Social History Review 47(2): 141-177.

\section{Chalmers, David}

2006 «Strong and weak emergence ", in Philip Clayton et Paul Davies (dir), The Re-emergence of Emergence. Oxford, Oxford University Press.

\section{Clark, William,}

Golinksi, Jan et Schaffer, Simon (éd.)

2001 The Sciences in Enlightened Europe. Chicago, University of Chicago Press.

\section{Cohen, Claudine}

2011 La Méthode de Zadig. La trace, le fossile, la preuve. Paris, Seuil.

\section{Cooren, François}

2013 Manière de faire parler. Interaction et ventriloquie,

Paris, Le Bord de l'Eau (édition originale Action and Agency in Dialogue: Passion, Incarnation and Ventriloquism. Amsterdam, John Benjamins Publishing Company, 2010).

\section{Daston, Lorraine} et Park, Katharine (éd.)

1998 Wonders and the Order of Nature, 1150-1750. New York, Zone Books.

\section{Edgeworth, Matt}

1992 "Analogy as practical reason: The perception of objects in archaeological practice ", Quarterly Newsletter of the Laboratory of Comparative Human Cognition 14(1): 3-5.

2003 Acts of Discovery: An Ethnography of Archaeological Practice. Oxford, ArchaeoPress.

$\mathbf{2 0 0 7}$ «Double-artefacts: Exploring the other side of material culture ", in Vitor Oliviera Jorge et Julian Thomas (éd.), Overcoming the Modern Invention of Material Culture, numéro spécial du Journal of Iberian Archaeology 9-10(89-96).

\section{Edgeworth, Matt (éd.)}

2006 Ethnographies of Archaeological Practice: Cultural Encounters, Material

Transformations. Lanham, AltaMira Press.

\section{Ginzburg, Carlo}

2010 «Traces. Racines d'un paradigme indiciaire ", in Mythes, emblèmes, traces; morphologie et histoire, nouvelle édition augmentée, revue par Martin Rueff. Lagrasse, Verdier (édition originale Miti emblemi spie. Morfologia e storia. Turin, Einaudi, 1986).

2010 Le Fil et les Traces. Vrai faux fictif. Lagrasse, Verdier.

\section{Goldblatt, David}

2006 Art and Ventriloquism. Londres, Routledge.

\section{Goodwin, Charles}

2003 "Pointing as situated practice ", in Sotaro Kita (dir.), Pointing: Where Language, Culture and Cognition meet. Hillsdale, Lawrence Erlbaum Associates: 217-241.

\section{Grimaud, Emmanuel}

2003 "Le trésor de Toutânkhamon: objets, guides et visiteurs au musée du Caire", Gradhiva 34 : 15-28.

2004 "La formation des énigmes en archéologie ou l'art de traiter les objets non identifiés ", in Annales de la Fondation Fyssen 19: 67-78.

2008 "Les dérives du scénario: script, logiciels d'écriture et modèles de conception aléatoires au cinéma ", in Brigitte Baptandier et Giordana Charuty (éd.), Du corps au texte, approches comparatives. Nanterre, Société d'ethnologie.

\section{Guha, Sudeshna}

2005 « Negotiating evidence: History, archaeology and the Indus civilisation ", Modern Asian Studies 39(2) : 399-426.

\section{Heider, Fritz et Simmel, Marianne}

1944 "An experimental study of apparent behavior ", American Journal of Psychology 57 : 243-259.

\section{Hocquard, Emmanuel}

2007 Une grammaire de Tanger. Marseille, CipM.

\section{Hodder, Ian}

1999 The Archaeological Process. An Introduction. Londres, Wiley-Blackwell.

\section{Huxley, Thomas Henry}

1880 "On the method of Zadig: Retrospective prophecy as a function of Science ", Popular Science Monthly 17.

\section{Ingold, Tim}

2011 Une brève histoire des lignes. Bruxelles, Zones sensibles (édition originale Lines: A Brief History. Londres, Routledge, 2007).

\section{Kanisza, Gaetano}

1997 La Grammaire du voir. Paris, Diderot (édition originale Organization in Vision: Essays on Gestalt Perception. New York, Praeger, 1979).

\section{Mus, Paul}

1964 «Un cinéma solide. L'intégration du temps dans l'art de l'Inde et dans l'art contemporain ", Arts asiatiques 10(1) : 21-34.

\section{Peirce, Charles Sanders}

2002 (1903) «Conférences de Harvard", in Pragmatisme et pragmaticisme. CEuvres philosophiques I. Paris, Editions du Cerf.

\section{Putnam, Hillary}

1981 "Brain in the Vat ", in Reason, Truth and History. Cambridge, Cambridge University Press.

\section{Schiffer, Michael Brian}

2002 Formation Processes of the Archaeological Record. Salt Lake City, University of Utah Press.

\section{Shanks, Michael}

1992 Experiencing the Past. On the Character of Archaeology. Londres, Routledge.

2001 Theatre/Archaeology. Disciplinary Dialogues. Londres, Routledge.

\section{Testart, Alain}

2006 "Comment concevoir une collaboration entre anthropologie sociale et archéologie? À quel prix? Et pourquoi? ", Bulletin de la Société préhistorique française 103(2): 385-395.

\section{Thouard, Denis (dir.)}

2007 L'Interprétation des indices: enquête sur le paradigme indiciaire avec Carlo Ginzburg. Villeneuve d'Ascq, Presses universitaires du Septentrion.

\section{Tilley, Christopher}

2004 The Materiality of Stone: Explorations in Landscape Phenomenology. Oxford, Berg. 


\section{Voisinat, Claudie (dir.)}

2008 Imaginaires archéologiques. Paris, Éditions de la Maison des sciences de l'homme, "Ethnologie de la France, cahier 22 ».

\section{Wheeler, Sir Mortimer}

1954 Archaeology from the Earth. Oxford, Clarendon Press.

\section{Willoughby, Dominique}

2008 Le Cinéma graphique.

Une histoire des dessins animés. Paris, Textuel.

\section{Witzel, Michael}

\section{et Farmer, Steve}

2000 "Horseplay in Harappa",

Frontline, 13 octobre.

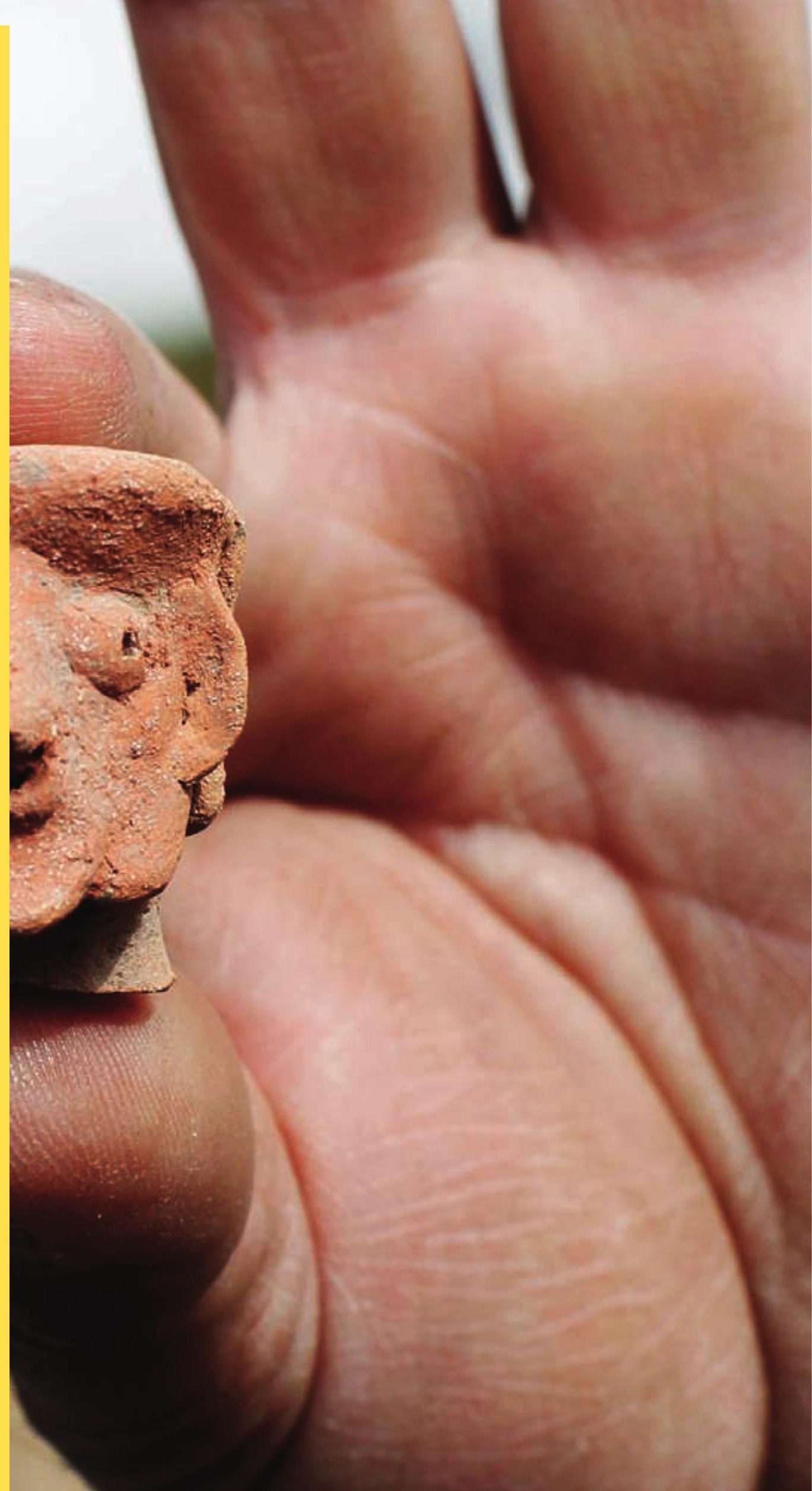

